Supporting information

\title{
Extreme Basicity of Biguanide Drugs in Aqueous Solutions: Ion Transfer Voltammetry and DFT Calculations
}

\author{
Jan Langmaier, ${ }^{a}$ Martin Pižl, a,b, Zdeněk Samec*a and Stanislav Záliš ${ }^{* a}$ \\ ${ }^{a} J$. Heyrovský Institute of Physical Chemistry of CAS, v.v.i, Dolejškova 3, 18223 Prague 8, \\ Czech Republic \\ ${ }^{b}$ Department of Inorganic Chemistry, University of Chemistry and Technology, Prague, \\ Technická 5, 16628 Prague 6, Czech Republic
}
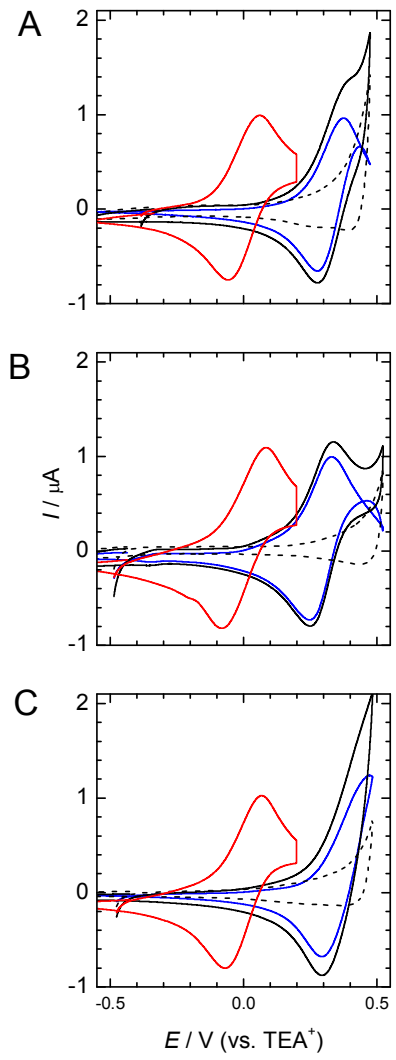

Fig. S1 Cyclic voltammograms of $0.2 \mathrm{mM} \mathrm{TEA}^{+}$(full red line) and $0.2 \mathrm{mM} \mathrm{MFH}^{+}$(full black line) measured at $\mathrm{pH} 13.8$ (A), 7.41 (B) and 2.05 (C) and the sweep rate $10 \mathrm{mV} \mathrm{s}^{-1}$. Full blue lines show the voltammograms corrected for the background current (dashed black line). 


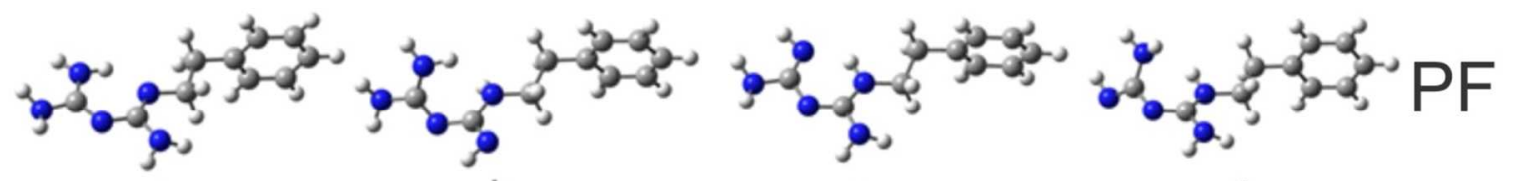

a

b

C

d
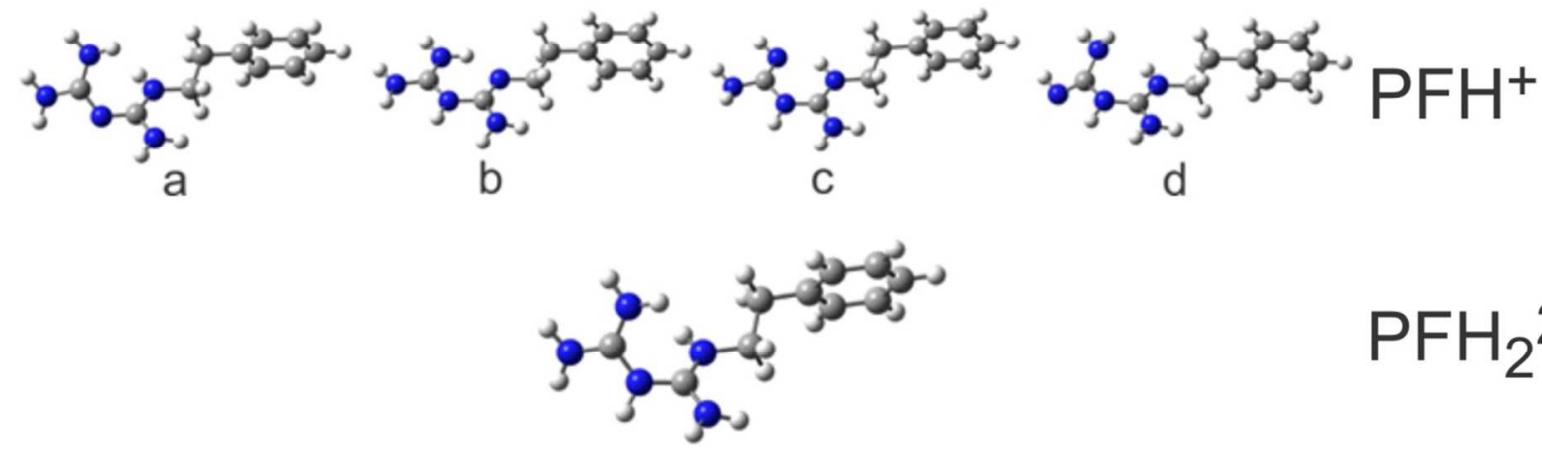

$\mathrm{PFH}_{2}{ }^{2+}$

Fig. S2 DFT optimized structures of the most stable tautomers of $\mathrm{PF}, \mathrm{PFH}^{+}$and $\mathrm{PFH}_{2}{ }^{2+}$.
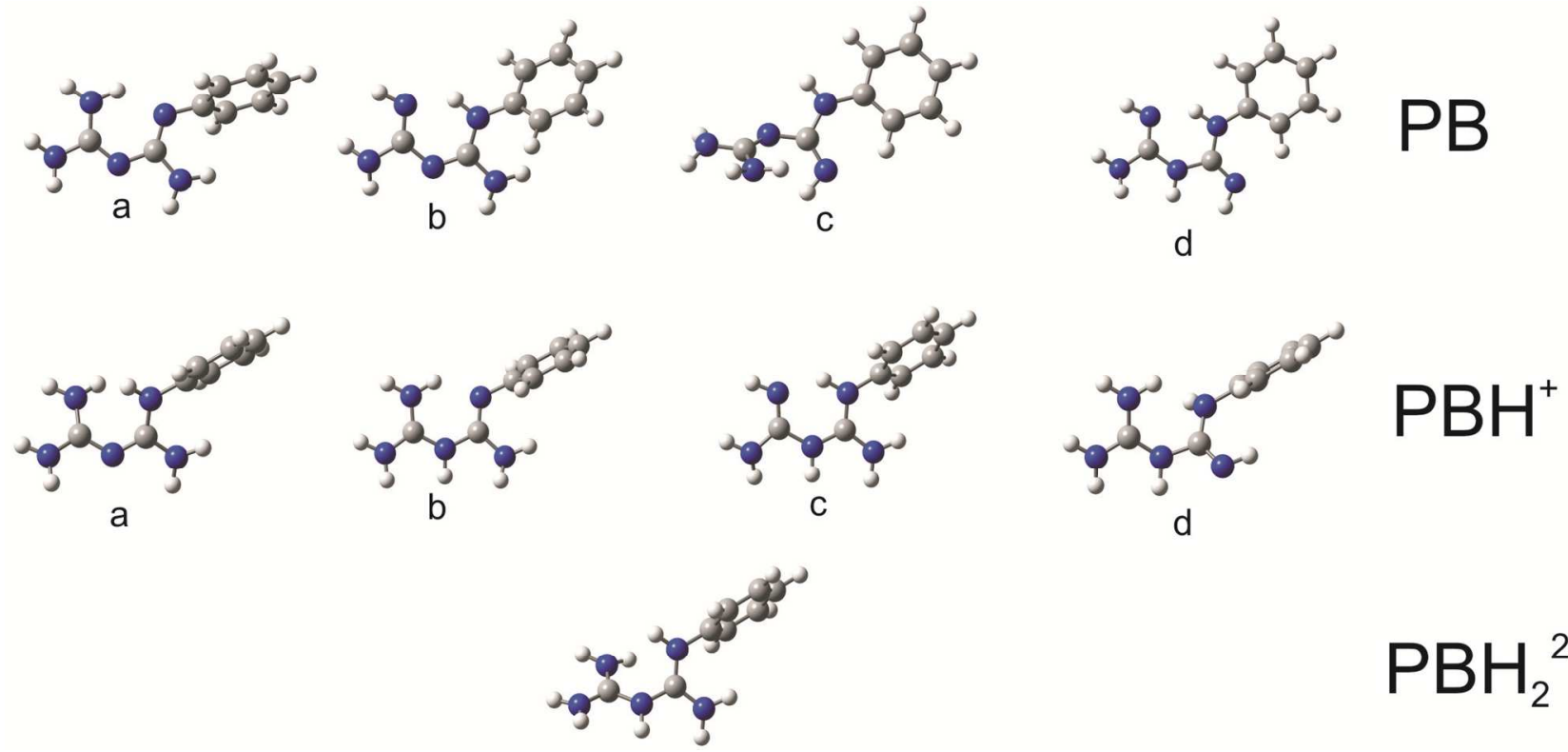

$\mathrm{PBH}_{2}{ }^{2+}$

Fig. S3 DFT optimized structures of the most stable tautomers of $\mathrm{PB}, \mathrm{PBH}^{+}$and $\mathrm{PBH}_{2}{ }^{2+}$. 

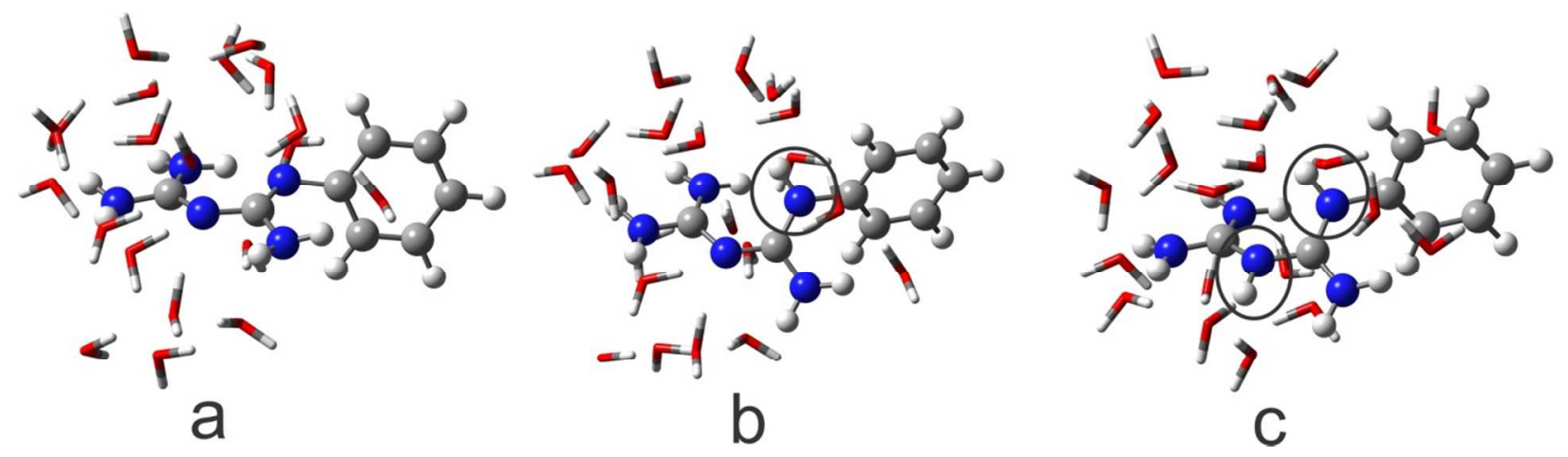

Fig. S4 DFT/M06-2X/G3/PCM optimized structure of (a) $\left\{(\mathrm{PB}) \cdot\left(\mathrm{H}_{2} \mathrm{O}\right)_{20}\right\}, \quad$ (b) $\left\{(\mathrm{PBH}) \cdot\left(\mathrm{H}_{2} \mathrm{O}\right)_{20}\right\}^{+}$and $(\mathrm{c})\left\{(\mathrm{PBH}) \cdot\left(\mathrm{H}_{2} \mathrm{O}\right)_{20}\right\}^{2+}$. The shortest hydrogen bond distances were calculated to be of $1.867 \AA$ for system (b) and $1.785 \AA$ and $1.798 \AA$ for system (c). The circles indicate the groups including protons in the mono- and di-protonated PB forms. 
Table S1 Relative free energies in $\mathrm{kcal} / \mathrm{mol}$ of the most stable tautomers a, b and c of MF and $\mathrm{MFH}^{+}$.

\begin{tabular}{lll}
\hline Tautomer & $\mathrm{MF}$ & $\mathrm{MFH}^{+}$ \\
\hline $\mathrm{a}$ & 0 & 0 \\
$\mathrm{~b}$ & 2.93 & 10.06 \\
$\mathrm{c}$ & 2.97 & 14.05 \\
\hline
\end{tabular}

Table S2 Relative free energies in $\mathrm{kcal} / \mathrm{mol}$ of the most stable tautomers a, b, c and d of PF and $\mathrm{PFH}^{+}$

\begin{tabular}{lll}
\hline Tautomer & $\mathrm{PF}$ & $\mathrm{PFH}^{+}$ \\
\hline $\mathrm{a}$ & 0 & 0 \\
$\mathrm{~b}$ & 0.13 & 6.24 \\
$\mathrm{c}$ & 0.19 & 7.19 \\
$\mathrm{~d}$ & 0.21 & 11.81 \\
\hline
\end{tabular}

Table S3 Relative free energies in $\mathrm{kcal} / \mathrm{mol}$ of the most stable tautomers of $\mathrm{PB}$ and $\mathrm{PFH}^{+}$

\begin{tabular}{lll}
\hline Tautomer & $\mathrm{PB}$ & $\mathrm{PBH}^{+}$ \\
\hline $\mathrm{a}$ & 0 & 0 \\
$\mathrm{~b}$ & 4.44 & 4.02 \\
$\mathrm{c}$ & 6.52 & 6.66 \\
$\mathrm{~d}$ & 9.88 & 11.82 \\
\hline
\end{tabular}


Table S4 The comparison of the experimental [8] and DFT calculated bond lengths $(\AA)$ and angles $\left({ }^{\circ}\right)$ of $\mathrm{MFH}^{+}$. For atom numbering see Chart 1.

\begin{tabular}{lll}
\hline bond & Experimental & Calculated \\
\hline N1-C2 & $1.332(3)$ & 1.338 \\
C2-N3 & $1.327(3)$ & 1.347 \\
C2-N4 & $1.339(2)$ & 1.323 \\
N4-C5 & $1.347(2)$ & 1.339 \\
C5-N6 & $1.333(3)$ & 1.350 \\
C5-N7 & $1.337(2)$ & 1.331 \\
N7-C8 & $1.451(3)$ & 1.460 \\
N7-C9 & $1.455(3)$ & 1.459 \\
\hline angle & & \\
\hline N1-C2-N4 & 118.0 & 117.0 \\
N3-C2-N4 & 124.6 & 125.4 \\
C2-N4-C5 & 121.8 & 123.5 \\
N4-C5-N7 & 118.2 & 119.0 \\
N4-C5-N6 & 122.3 & 122.5 \\
N7-C5-N6 & 119.3 & 118.5 \\
N1-C2-N3 & 117.3 & 117.6 \\
\hline
\end{tabular}


Table S5 The comparison of the experimental [9] and DFT calculated bond lengths ( $\AA$ ) and angles $\left({ }^{\circ}\right)$ of $\mathrm{MFH}^{+} \mathrm{Cl}^{-}$. For atom numbering see Chart 1.

\begin{tabular}{lll}
\hline bond & Experimental & Calculated \\
\hline N1-C2 & $1.335(4)$ & 1.337 \\
C2-N3 & $1.331(5)$ & 1.341 \\
C2-N4 & $1.332(4)$ & 1.329 \\
N4-C5 & $1.348(3)$ & 1.337 \\
C5-N6 & $1.330(4)$ & 1.343 \\
C5-N7 & $1.334(3)$ & 1.341 \\
N7-C8 & $1.461(4)$ & 1.461 \\
N7-C9 & $1.461(4)$ & 1.461 \\
\hline angle & & \\
\hline N1-C2-N4 & 118.0 & 117.9 \\
N3-C2-N4 & 124.0 & 125.0 \\
C2-N4-C5 & 122.5 & 122.2 \\
N4-C5-N7 & 122.7 & 124.5 \\
N4-C5-N6 & 116.7 & 117.0 \\
N7-C5-N6 & 120.2 & 118.5 \\
N1-C2-N3 & 118.0 & 117.1 \\
\hline
\end{tabular}


Table S6 The comparison of the experimental and DFT calculated bond lengths $(\AA)$ and angles $\left({ }^{\circ}\right)$ of PB. For atom numbering see Chart 1.

\begin{tabular}{lll}
\hline Bond & Experimental & Calculated \\
\hline N1-C2 & 1.344 & 1.365 \\
C2-N3 & 1.325 & 1.342 \\
C2-N4 & 1.329 & 1.315 \\
N4-C5 & 1.380 & 1.374 \\
C5-N6 & 1.371 & 1.376 \\
C5-N7 & 1.289 & 1.301 \\
N7-C8 & 1.405 & 1.407 \\
\hline Angle & & \\
\hline N1-C2-N4 & 117.1 & 116.7 \\
N3-C2-N4 & 124.9 & 126.5 \\
C2-N4-C5 & 121.1 & 121.8 \\
N4-C5-N7 & 124.8 & 125.1 \\
N4-C5-N6 & 110.8 & 111.6 \\
N7-C5-N6 & 124.3 & 123.2 \\
N1-C2-N3 & 118.0 & 116.8 \\
\hline
\end{tabular}


Table S7 The comparison of the experimental and DFT calculated bond lengths $(\AA)$ and angles $\left({ }^{\circ}\right)$ of $\mathrm{PBH}^{+}$. For atom numbering see Chart 1.

\begin{tabular}{lll}
\hline Bond & Experimental $\mathrm{PBH}^{+} \mathrm{Cl}^{-}$ & Calculated $\mathrm{PBH}^{+}$ \\
\hline $\mathrm{N} 1-\mathrm{C} 2$ & 1.332 & 1.334 \\
$\mathrm{C} 2-\mathrm{N} 3$ & 1.333 & 1.345 \\
$\mathrm{C} 2-\mathrm{N} 4$ & 1.336 & 1.331 \\
$\mathrm{~N} 4-\mathrm{C} 5$ & 1.335 & 1.330 \\
$\mathrm{C} 5-\mathrm{N} 6$ & 1.337 & 1.332 \\
$\mathrm{C} 5-\mathrm{N} 7$ & 1.357 & 1.353 \\
N7-C8 & 1.422 & 1.429 \\
\hline Angle & & \\
\hline N1-C2-N4 & 117.6 & 116.8 \\
N3-C2-N4 & 124.2 & 125.1 \\
C2-N4-C5 & 122.1 & 123.8 \\
N4-C5-N7 & 118.2 & 124.0 \\
N4-C5-N6 & 125.0 & 117.2 \\
N7-C5-N6 & 116.8 & 118.8 \\
N1-C2-N3 & 118.1 & 118.1 \\
\hline
\end{tabular}


Table S8 The comparison of the experimental and DFT calculated bond lengths $(\AA)$ and angles $\left(^{\circ}\right)$ of $\mathrm{PBH}^{+} \mathrm{Cl}^{-}$. For atom numbering see Chart 1.

\begin{tabular}{lll}
\hline Bond & Experimental $\mathrm{PBH}^{+} \mathrm{Cl}^{-}$ & Calculated $\mathrm{PBH}^{+} \mathrm{Cl}^{-}$ \\
\hline N1-C2 & 1.332 & 1.336 \\
C2-N3 & 1.333 & 1.344 \\
C2-N4 & 1.336 & 1.328 \\
N4-C5 & 1.335 & 1.328 \\
C5-N6 & 1.337 & 1.350 \\
C5-N7 & 1.357 & 1.342 \\
N7-C8 & 1.422 & 1.415 \\
\hline Angle & & 116.9 \\
\hline N1-C2-N4 & 117.6 & 125.0 \\
N3-C2-N4 & 124.2 & 123.6 \\
C2-N4-C5 & 122.1 & 119.5 \\
N4-C5-N7 & 118.2 & 124.7 \\
N4-C5-N6 & 125.0 & 115.8 \\
N7-C5-N6 & 116.8 & 118.1 \\
N1-C2-N3 & 118.1 & \\
\hline
\end{tabular}


Table S9. Cartesian coordinates corresponding to the optimized structures of all MF and MFH+ under study.

$\begin{array}{lrrr}\text { MF - tautomer a } & & \\ \mathrm{N} & 1.69251900 & 0.24476800 & -0.17594400 \\ \mathrm{~N} & -0.59555300 & 0.52710600 & -0.15436500 \\ \mathrm{~N} & 0.30091900 & -1.65817000 & 0.01171200 \\ \mathrm{~N} & -2.79839300 & 1.11820700 & 0.01162300 \\ \mathrm{~N} & -2.27846100 & -1.12649200 & 0.11765500 \\ \mathrm{C} & 1.86976100 & 1.64199600 & 0.20092500 \\ \mathrm{C} & 2.88087100 & -0.57643100 & -0.03420400 \\ \mathrm{C} & 0.45400200 & -0.36769600 & -0.08456200 \\ \mathrm{C} & -1.84008700 & 0.13698800 & -0.01898100 \\ \mathrm{H} & 0.97732100 & 2.20556600 & -0.04613500 \\ \mathrm{H} & 2.71877500 & 2.05141200 & -0.34842100 \\ \mathrm{H} & 2.07029100 & 1.74275900 & 1.27523100 \\ \mathrm{H} & 3.75440200 & 0.04112400 & -0.23710100 \\ \mathrm{H} & 2.87783100 & -1.39449100 & -0.75693400 \\ \mathrm{H} & 2.98250100 & -0.99555400 & 0.97578600 \\ \mathrm{H} & 1.18539400 & -2.14336000 & 0.10120500 \\ \mathrm{H} & -3.73603400 & 0.87231900 & -0.26977700 \\ \mathrm{H} & -2.48126000 & 2.02101700 & -0.30787200 \\ \mathrm{H} & -1.54966500 & -1.83688300 & 0.18333000 \\ \mathrm{H} & -3.23405600 & -1.31098000 & 0.37684700 \\ \mathrm{MF}-\text { tautomer } & & & \\ \mathrm{N} & 1.66415000 & 0.20723300 & 0.13762900 \\ \mathrm{~N} & -0.60268500 & 0.56738300 & 0.11471200 \\ \mathrm{~N} & 0.27623800 & -1.60466200 & -0.28549100 \\ \mathrm{~N} & -2.76426500 & 0.96055700 & -0.54927800 \\ \mathrm{~N} & -2.40321000 & -0.92497400 & 0.71789700 \\ \mathrm{C} & 1.86011600 & 1.64395300 & 0.28272100 \\ \mathrm{C} & 2.871614000 & -0.61360700 & -0.20881900 \\ \mathrm{C} & 0.39947500 & -0.26244700 & -0.01693000 \\ \mathrm{H} & -1.90843600 & 0.10720700 & 0.11632800 \\ \mathrm{H} & 1.71145800 & 2.16751200 & -0.66715600 \\ \mathrm{H} & 2.87603600 & 1.81829000 & 0.63149500 \\ \mathrm{H} & 2.71602500 & -0.09940500 & 0.11939000 \\ \mathrm{H} & -0.78544900 & -1.28822500 \\ \mathrm{H} & -1.57314400 & 0.30912800\end{array}$




$\begin{array}{rrrr}H & 1.03116100 & -2.06544600 & -0.76925200 \\ H & -3.68266200 & 0.59021800 & -0.74245200 \\ H & -2.35293500 & 1.51505400 & -1.28421700 \\ H & -1.67998800 & -1.38818400 & 1.26190000 \\ H & -0.64393300 & -1.91091000 & -0.56925800\end{array}$

$\begin{array}{lrrr}\text { MF - tautomer c } & & \\ \mathrm{N} & 1.66157100 & 0.21409300 & -0.02315100 \\ \mathrm{~N} & -0.58836000 & 0.63585700 & 0.11077500 \\ \mathrm{~N} & 0.21581000 & -1.55260400 & -0.38349800 \\ \mathrm{~N} & -2.80886000 & 0.95695500 & -0.61148100 \\ \mathrm{~N} & -2.31633700 & -0.89034900 & 0.67642800 \\ \mathrm{C} & 1.91851100 & 1.61363700 & 0.28535600 \\ \mathrm{C} & 2.77555200 & -0.72461500 & -0.05180000 \\ \mathrm{C} & 0.37844500 & -0.22035800 & -0.09725600 \\ \mathrm{C} & -1.92125200 & 0.27326900 & 0.02003100 \\ \mathrm{H} & 1.25096500 & 2.24863300 & -0.29369800 \\ \mathrm{H} & 2.94910000 & 1.84220100 & 0.02107100 \\ \mathrm{H} & 1.76326200 & 1.82721100 & 1.34721500 \\ \mathrm{H} & 3.67257900 & -0.20683400 & 0.27814400 \\ \mathrm{H} & 2.96042200 & -1.10887800 & -1.06007300 \\ \mathrm{H} & 2.59925200 & -1.56511300 & 0.62292800 \\ \mathrm{H} & 0.93008800 & -2.02008800 & -0.91985800 \\ \mathrm{H} & -2.33911500 & 1.72629100 & -1.08304100 \\ \mathrm{H} & -1.79383300 & -1.11444300 & 1.51268400 \\ \mathrm{H} & -3.31681200 & -0.96332300 & 0.80133000 \\ \mathrm{H} & -0.73022500 & -1.86493400 & -0.54819000\end{array}$

\begin{tabular}{crrc}
\multicolumn{4}{l}{ MFH+ - tautomer a } \\
$\mathrm{N}$ & 1.65816600 & 0.26647800 & 0.02364200 \\
$\mathrm{~N}$ & -0.60641500 & 0.52907000 & -0.29647700 \\
$\mathrm{~N}$ & 0.38122100 & -1.61088100 & -0.37305400 \\
$\mathrm{~N}$ & -2.84002800 & 0.90938000 & -0.43295800 \\
$\mathrm{~N}$ & -2.15145200 & -0.92131200 & 0.77174600 \\
$\mathrm{C}$ & 1.81616100 & 1.67357300 & 0.38299300 \\
$\mathrm{C}$ & 2.87644100 & -0.53454200 & -0.04921300 \\
$\mathrm{C}$ & 0.46178000 & -0.27389100 & -0.20105200 \\
$\mathrm{C}$ & -1.83458300 & 0.15054400 & 0.01949800 \\
$\mathrm{H}$ & 0.84080500 & 2.13520600 & 0.48845900 \\
$\mathrm{H}$ & 2.38463900 & 2.18686400 & -0.39440500 \\
$\mathrm{H}$ & 2.35867000 & 1.73980900 & 1.32714700
\end{tabular}




$\begin{array}{lrrr}\mathrm{H} & 3.72724400 & 0.13962900 & 0.00431000 \\ \mathrm{H} & 2.92982800 & -1.07346900 & -0.99691800 \\ \mathrm{H} & 2.93999100 & -1.23847700 & 0.78430400 \\ \mathrm{H} & 1.20327200 & -2.19310700 & -0.38993500 \\ \mathrm{H} & -2.61558900 & 1.70402500 & -1.00968700 \\ \mathrm{H} & -1.42962600 & -1.40391700 & 1.28449300 \\ \mathrm{H} & -3.10036300 & -1.06182400 & 1.08382600 \\ \mathrm{H} & -0.45120300 & -2.00021400 & -0.78915100 \\ \mathrm{H} & -3.79691100 & 0.76222000 & -0.15609400\end{array}$

$\begin{array}{crrr}\text { MFH+ - tautomer b } & & \\ \mathrm{N} & -1.69472300 & 0.26364500 & -0.13436300 \\ \mathrm{~N} & 0.61151200 & 0.42178900 & -0.28278700 \\ \mathrm{~N} & -0.33119300 & -1.67926500 & 0.00532600 \\ \mathrm{~N} & 2.81124000 & 1.07362300 & -0.17825500 \\ \mathrm{~N} & 2.25086000 & -1.10705900 & 0.33113800 \\ \mathrm{C} & -1.86612000 & 1.59357000 & 0.45482100 \\ \mathrm{C} & -2.91937300 & -0.51934900 & -0.20990000 \\ \mathrm{C} & -0.51463700 & -0.41645800 & -0.09765900 \\ \mathrm{C} & 1.90705900 & 0.10928200 & -0.03477500 \\ \mathrm{H} & -0.91059800 & 2.04150700 & 0.71448600 \\ \mathrm{H} & -2.45571700 & 1.51078800 & 1.37211800 \\ \mathrm{H} & -2.38995700 & 2.24463600 & -0.24593600 \\ \mathrm{H} & -3.72655700 & 0.14460900 & -0.51606000 \\ \mathrm{H} & -3.18047500 & -0.96429800 & 0.75710900 \\ \mathrm{H} & -2.81681100 & -1.30477900 & -0.95851500 \\ \mathrm{H} & -1.18737400 & -2.17305500 & 0.23378300 \\ \mathrm{H} & 2.53902300 & 2.03148800 & -0.33579500 \\ \mathrm{H} & 1.51384300 & -1.81624200 & 0.32836400 \\ \mathrm{H} & 0.44256500 & 1.31352300 & -0.72787800 \\ \mathrm{H} & 3.79546700 & 0.87682400 & -0.08040500 \\ \mathrm{H} & 3.20114300 & -1.31640200 & 0.59639700\end{array}$

$\mathrm{MFH}+$ - tautomer c

$\begin{array}{lrrr}\mathrm{N} & 1.67607600 & 0.19399200 & 0.02967900 \\ \mathrm{~N} & -0.60374000 & 0.57310800 & 0.01045700 \\ \mathrm{~N} & 0.24051300 & -1.58738600 & -0.28746500 \\ \mathrm{~N} & -2.85105300 & 0.93302100 & -0.64922100 \\ \mathrm{~N} & -2.31117500 & -0.88619500 & 0.68681800 \\ \mathrm{C} & 1.92597300 & 1.57534000 & 0.44997600 \\ \mathrm{C} & 2.82752500 & -0.65511700 & -0.28406100\end{array}$




$\begin{array}{lrrr}\mathrm{C} & 0.44827400 & -0.29126500 & -0.07081100 \\ \mathrm{C} & -1.98962100 & 0.25059700 & -0.01905600 \\ \mathrm{H} & 1.88833200 & 2.25912500 & -0.40099600 \\ \mathrm{H} & 2.91804400 & 1.61491100 & 0.89189000 \\ \mathrm{H} & 1.20540000 & 1.87546000 & 1.20918300 \\ \mathrm{H} & 3.67923600 & -0.00892100 & -0.47653200 \\ \mathrm{H} & 2.63151700 & -1.23772200 & -1.18424800 \\ \mathrm{H} & 3.06462000 & -1.31805800 & 0.55109900 \\ \mathrm{H} & 0.98145100 & -2.25452200 & -0.13636300 \\ \mathrm{H} & -2.42082000 & 1.65502900 & -1.22177400 \\ \mathrm{H} & -1.85869700 & -0.99405500 & 1.58684500 \\ \mathrm{H} & -0.39932200 & 1.53707000 & -0.21805000 \\ \mathrm{H} & -3.30794600 & -1.05482500 & 0.72162800 \\ \mathrm{H} & -0.70905700 & -1.93660400 & -0.31084200\end{array}$


Table S10. Cartesian coordinates corresponding to the optimized structures of all PF and PFH+ under study.

$\begin{array}{lrrr}\text { PF - tautomer a } & & \\ \mathrm{N} & 1.28811300 & -0.01592700 & 0.06730400 \\ \mathrm{~N} & 3.53501500 & 0.76569800 & 0.01582200 \\ \mathrm{~N} & 1.82420800 & 2.27684700 & -0.00207900 \\ \mathrm{~N} & 5.43707400 & -0.49451000 & -0.15753400 \\ \mathrm{~N} & 3.41892900 & -1.60144600 & 0.02750600 \\ \mathrm{C} & -0.99228900 & -0.89251300 & -0.00590500 \\ \mathrm{C} & -2.45712100 & -0.53854900 & -0.01538200 \\ \mathrm{C} & -0.11311600 & 0.35848100 & 0.06263000 \\ \mathrm{C} & -3.16730200 & -0.40366700 & 1.18005400 \\ \mathrm{C} & -3.12198900 & -0.28504700 & -1.21765800 \\ \mathrm{C} & -4.50800900 & -0.02799900 & 1.17615500 \\ \mathrm{C} & -4.46247800 & 0.09092900 & -1.22741000 \\ \mathrm{C} & -5.16006200 & 0.22048500 & -0.02900300 \\ \mathrm{C} & 2.16393500 & 0.93640900 & 0.04566900 \\ \mathrm{C} & 4.07438700 & -0.42951800 & -0.02490900 \\ \mathrm{H} & -0.76170500 & -1.52860900 & 0.85291100 \\ \mathrm{H} & -0.73207600 & -1.45055700 & -0.90933800 \\ \mathrm{H} & -0.35220300 & 1.00695700 & -0.79372600 \\ \mathrm{H} & -0.38281300 & 0.92159700 & 0.97015300 \\ \mathrm{H} & -2.66389600 & -0.59931300 & 2.12186900 \\ \mathrm{H} & -2.58281700 & -0.38765500 & -2.15442500 \\ \mathrm{H} & -5.04416600 & 0.06752200 & 2.11335600 \\ \mathrm{H} & -4.96297700 & 0.27948100 & -2.17021300 \\ \mathrm{H} & -6.20427300 & 0.50999700 & -0.03447800 \\ \mathrm{H} & 2.57652000 & 2.90810800 & 0.22871800 \\ \mathrm{H} & 5.91853900 & 0.37037600 & 0.03717900 \\ \mathrm{H} & 2.40130100 & -1.53842200 & 0.02054700 \\ \mathrm{H} & 0.92242200 & 2.54731700 & 0.35963100 \\ \mathrm{H} & 5.90561600 & -1.32612100 & 0.17023600 \\ \mathrm{H} & 3.90341800 & -2.46936700 & -0.13499600\end{array}$

PF - tautomer b

$\begin{array}{lrrr}\mathrm{N} & 1.24187900 & 0.17970100 & 0.20210700 \\ \mathrm{~N} & 3.49824300 & 0.83774100 & 0.09976400 \\ \mathrm{~N} & 1.90311000 & 1.65787000 & -1.44943900 \\ \mathrm{~N} & 5.31671400 & -0.39327100 & 0.76847800 \\ \mathrm{~N} & 3.61860700 & -1.48469000 & -0.36409100\end{array}$




$\begin{array}{lrrr}\mathrm{C} & -1.03156700 & -0.57401300 & 0.69813700 \\ \mathrm{C} & -2.49240200 & -0.36776100 & 0.38627600 \\ \mathrm{C} & -0.16317600 & 0.38834400 & -0.11220100 \\ \mathrm{C} & -3.25011500 & 0.55897400 & 1.10550700 \\ \mathrm{C} & -3.10151000 & -1.06230200 & -0.66094200 \\ \mathrm{C} & -4.58633800 & 0.78639800 & 0.78798100 \\ \mathrm{C} & -4.43750800 & -0.83839400 & -0.98206400 \\ \mathrm{C} & -5.18399200 & 0.08762000 & -0.25776000 \\ \mathrm{C} & 2.21189600 & 0.92549500 & -0.44070300 \\ \mathrm{C} & 4.09103200 & -0.31253600 & 0.16034500 \\ \mathrm{H} & -0.85484200 & -0.41035200 & 1.76571100 \\ \mathrm{H} & -0.73691700 & -1.60107200 & 0.46703400 \\ \mathrm{H} & -0.30736000 & 0.21267600 & -1.18019200 \\ \mathrm{H} & -0.46168900 & 1.42502000 & 0.08813800 \\ \mathrm{H} & -2.78833800 & 1.10374100 & 1.92311500 \\ \mathrm{H} & -2.52343800 & -1.78674300 & -1.22620400 \\ \mathrm{H} & -5.16108800 & 1.50679300 & 1.35842900 \\ \mathrm{H} & -4.89607300 & -1.38871600 & -1.79545400 \\ \mathrm{H} & -6.22465800 & 0.26141800 & -0.50465600 \\ \mathrm{H} & 2.75863800 & 2.07515600 & -1.80886600 \\ \mathrm{H} & 5.93268400 & -1.13984500 & 0.48135000 \\ \mathrm{H} & 5.77401200 & 0.49604000 & 0.90503700 \\ \mathrm{H} & 2.62034000 & -1.53187900 & -0.51679900 \\ \mathrm{H} & 4.03472000 & -2.34397800 & -0.03686000 \\ \mathrm{H} & 1.44621000 & -0.01066200 & 1.17503100\end{array}$

$\begin{array}{lrrr}\mathrm{PF}-\text { tautomer c } & & \\ \mathrm{N} & 1.30267400 & 0.09674600 & 0.01478600 \\ \mathrm{~N} & 3.58496000 & 0.74025400 & 0.04214200 \\ \mathrm{~N} & 1.92858500 & 2.31999100 & -0.03092900 \\ \mathrm{~N} & 5.13186700 & -0.83349300 & 0.67232200 \\ \mathrm{~N} & 3.63705400 & -1.45941700 & -0.95941700 \\ \mathrm{C} & -0.94856600 & -0.83948300 & -0.11593300 \\ \mathrm{C} & -2.42118600 & -0.52247800 & -0.04407700 \\ \mathrm{C} & -0.11406800 & 0.42398300 & 0.09321600 \\ \mathrm{C} & -3.09728700 & -0.55524700 & 1.17701700 \\ \mathrm{C} & -3.12213400 & -0.14527400 & -1.19120700 \\ \mathrm{C} & -4.44625500 & -0.21960400 & 1.25138600 \\ \mathrm{C} & -4.47107600 & 0.19089400 & -1.12113600 \\ \mathrm{C} & -5.13671900 & 0.15460200 & 0.10148400 \\ \mathrm{C} & 2.30611400 & 1.00649200 & 0.01797100\end{array}$




$\begin{array}{lrrr}\mathrm{C} & 4.04041000 & -0.56007900 & -0.12326200 \\ \mathrm{H} & -0.68650900 & -1.57314000 & 0.65178600 \\ \mathrm{H} & -0.70119000 & -1.27050900 & -1.08944900 \\ \mathrm{H} & -0.36024700 & 1.15098900 & -0.68617000 \\ \mathrm{H} & -0.36340300 & 0.86695000 & 1.06516600 \\ \mathrm{H} & -2.56277900 & -0.84991500 & 2.07469800 \\ \mathrm{H} & -2.60666500 & -0.11865800 & -2.14617600 \\ \mathrm{H} & -4.95837200 & -0.25371500 & 2.20590400 \\ \mathrm{H} & -5.00262500 & 0.47774700 & -2.02097300 \\ \mathrm{H} & -6.18747000 & 0.41323000 & 0.15729900 \\ \mathrm{H} & 2.68034700 & 2.97103400 & 0.13940500 \\ \mathrm{H} & 5.20493700 & -0.30211300 & 1.52606900 \\ \mathrm{H} & 2.90877300 & -1.07631700 & -1.55660900 \\ \mathrm{H} & 1.57107300 & -0.86969200 & 0.14015800 \\ \mathrm{H} & 1.03794100 & 2.58820700 & 0.35950300 \\ \mathrm{H} & 5.39481700 & -1.80549900 & 0.73431200\end{array}$

$\begin{array}{lrrr}\text { PF - tautomer d } & & \\ \mathrm{N} & 1.34902500 & 0.11221300 & 0.10274300 \\ \mathrm{~N} & 3.61722200 & 0.76274000 & 0.11601900 \\ \mathrm{~N} & 1.95454600 & 2.34867200 & 0.01581300 \\ \mathrm{~N} & 4.65493200 & -1.21453300 & 0.91686400 \\ \mathrm{~N} & 3.83103000 & -1.14872400 & -1.23483000 \\ \mathrm{C} & -0.89682900 & -0.82913800 & -0.09579100 \\ \mathrm{C} & -2.37182100 & -0.51781600 & -0.05190000 \\ \mathrm{C} & -0.07114600 & 0.43303300 & 0.15387400 \\ \mathrm{C} & -3.07654300 & -0.57741500 & 1.15190100 \\ \mathrm{C} & -3.04693500 & -0.11894500 & -1.20723600 \\ \mathrm{C} & -4.42800500 & -0.24711000 & 1.20128600 \\ \mathrm{C} & -4.39823700 & 0.21196100 & -1.16219900 \\ \mathrm{C} & -5.09245200 & 0.14868400 & 0.04336000 \\ \mathrm{C} & 2.34866900 & 1.03734300 & 0.09825300 \\ \mathrm{C} & 4.02806400 & -0.56665600 & 0.00126100 \\ \mathrm{H} & -0.65109200 & -1.57550300 & 0.66524700 \\ \mathrm{H} & -0.62463600 & -1.24267800 & -1.07008400 \\ \mathrm{H} & -0.29585000 & 1.16987600 & -0.62159600 \\ \mathrm{H} & -0.34857500 & 0.86242700 & 1.12469500 \\ \mathrm{H} & -2.56240900 & -0.88888600 & 2.05579100 \\ \mathrm{H} & -2.50921300 & -0.07119600 & -2.14904200 \\ \mathrm{H} & -4.96232700 & -0.30228700 & 2.14256700 \\ \mathrm{H} & -4.90944800 & 0.51566400 & -2.06828200\end{array}$




$\begin{array}{rrrr}\mathrm{H} & -6.14507600 & 0.40315900 & 0.07963600 \\ \mathrm{H} & 2.71005400 & 3.00510500 & 0.14805300 \\ \mathrm{H} & 4.74654200 & -0.61021000 & 1.72972000 \\ \mathrm{H} & 3.10278700 & -0.76575200 & -1.81840900 \\ \mathrm{H} & 3.92695400 & -2.15295600 & -1.26957600 \\ \mathrm{H} & 1.61323700 & -0.82704400 & 0.36730300 \\ \mathrm{H} & 1.09317500 & 2.61406400 & 0.47085800\end{array}$

PFH+ - tautomer a

$\begin{array}{lrrr}\mathrm{N} & 1.24780500 & 0.13944800 & -0.19137800 \\ \mathrm{~N} & 3.53539400 & 0.75415800 & -0.07761700 \\ \mathrm{~N} & 1.90380900 & 2.23494800 & 0.49037600 \\ \mathrm{~N} & 5.27383000 & -0.63563400 & -0.52382400 \\ \mathrm{~N} & 3.45860000 & -1.53101100 & 0.56273000 \\ \mathrm{C} & -1.00341200 & -0.81717000 & -0.27713000 \\ \mathrm{C} & -2.47427500 & -0.50845300 & -0.14564000 \\ \mathrm{C} & -0.17557700 & 0.45200700 & -0.08230400 \\ \mathrm{C} & -3.08781400 & -0.51395000 & 1.10855100 \\ \mathrm{C} & -3.23328400 & -0.16781500 & -1.26625700 \\ \mathrm{C} & -4.43401600 & -0.18677900 & 1.24112100 \\ \mathrm{C} & -4.58035800 & 0.15952600 & -1.13747200 \\ \mathrm{C} & -5.18376400 & 0.15113100 & 0.11719400 \\ \mathrm{C} & 2.23169800 & 1.00874900 & 0.07542000 \\ \mathrm{C} & 4.05506100 & -0.46452200 & -0.00470700 \\ \mathrm{H} & -0.70272200 & -1.55508600 & 0.47097500 \\ \mathrm{H} & -0.79551100 & -1.23542500 & -1.26582400 \\ \mathrm{H} & -0.45968000 & 1.20023500 & -0.83021300 \\ \mathrm{H} & -0.36982600 & 0.86257700 & 0.91246300 \\ \mathrm{H} & -2.50672300 & -0.78065200 & 1.98582400 \\ \mathrm{H} & -2.76641700 & -0.16306900 & -2.24607400 \\ \mathrm{H} & -4.89781200 & -0.19890600 & 2.22047100 \\ \mathrm{H} & -5.15849000 & 0.41803100 & -2.01685600 \\ \mathrm{H} & -6.23265500 & 0.40335700 & 0.21831900 \\ \mathrm{H} & 2.65272400 & 2.87846300 & 0.69273400 \\ \mathrm{H} & 5.71506300 & 0.14955100 & -0.97531500 \\ \mathrm{H} & 1.50420900 & -0.70672900 & -0.68276100 \\ \mathrm{H} & 0.96007000 & 2.58598400 & 0.46413800 \\ \mathrm{H} & 5.79986600 & -1.48568000 & -0.40021500 \\ \mathrm{H} & 3.97285600 & -2.39003200 & 0.68972500 \\ \mathrm{H} & 2.66342700 & -1.39232200 & 1.16795000\end{array}$


$\mathrm{PFH}+$ - tautomer b

$\begin{array}{lrrr}\mathrm{N} & 1.25102000 & 0.03310800 & -0.05239200 \\ \mathrm{~N} & 3.48632300 & 0.68581200 & -0.02581400 \\ \mathrm{~N} & 1.84836000 & 2.31684900 & 0.00118600 \\ \mathrm{~N} & 5.38524400 & -0.60445400 & 0.07509900 \\ \mathrm{~N} & 3.31470000 & -1.62798300 & 0.00371900 \\ \mathrm{C} & -1.01773500 & -0.87302200 & -0.00042900 \\ \mathrm{C} & -2.48621800 & -0.53237000 & 0.01020800 \\ \mathrm{C} & -0.15872900 & 0.39085500 & -0.05016900 \\ \mathrm{C} & -3.15045500 & -0.28660500 & 1.21413500 \\ \mathrm{C} & -3.19793600 & -0.40506000 & -1.18464500 \\ \mathrm{C} & -4.49462100 & 0.07532000 & 1.22564600 \\ \mathrm{C} & -4.54250800 & -0.04361000 & -1.17829500 \\ \mathrm{C} & -5.19480300 & 0.19770400 & 0.02802300 \\ \mathrm{C} & 2.10418100 & 0.97872100 & -0.04532900 \\ \mathrm{C} & 4.05777500 & -0.54298300 & 0.02006400 \\ \mathrm{H} & -0.75274700 & -1.43856400 & 0.89659700 \\ \mathrm{H} & -0.77741500 & -1.49375800 & -0.86733300 \\ \mathrm{H} & -0.41676400 & 0.96284300 & -0.95260200 \\ \mathrm{H} & -0.39701200 & 1.02273100 & 0.81632800 \\ \mathrm{H} & -2.60896700 & -0.38447300 & 2.15002800 \\ \mathrm{H} & -2.69394200 & -0.59528000 & -2.12714800 \\ \mathrm{H} & -4.99585300 & 0.25828200 & 2.16905700 \\ \mathrm{H} & -5.08114500 & 0.04641300 & -2.11451100 \\ \mathrm{H} & -6.24194800 & 0.47625300 & 0.03512000 \\ \mathrm{H} & 2.56994700 & 2.97740200 & -0.24635800 \\ \mathrm{H} & 5.95291000 & 0.22814700 & 0.10830100 \\ \mathrm{H} & 0.91335600 & 2.61520300 & -0.23142300 \\ \mathrm{H} & 5.85915800 & -1.49410000 & 0.10463200 \\ \mathrm{H} & 3.73642800 & -2.54298000 & 0.04986200 \\ \mathrm{H} & 2.29879200 & -1.48629800 & -0.02758100 \\ \mathrm{H} & 4.12195800 & 1.47114800 & -0.01080300\end{array}$

$\mathrm{PFH}+$ - tautomer c

$\begin{array}{lrrr}\mathrm{N} & 1.24473200 & 0.11767500 & -0.06027800 \\ \mathrm{~N} & 3.51110200 & 0.65389800 & -0.01541000 \\ \mathrm{~N} & 1.92190600 & 2.32172300 & 0.01094800 \\ \mathrm{~N} & 5.38145200 & -0.68003100 & 0.07222200 \\ \mathrm{~N} & 3.22252400 & -1.64979500 & -0.11222200 \\ \mathrm{C} & -1.00084900 & -0.84123200 & -0.02595700 \\ \mathrm{C} & -2.47435300 & -0.51829200 & -0.00256200\end{array}$




$\begin{array}{lrrr}\mathrm{C} & -0.17823600 & 0.44592200 & -0.03457900 \\ \mathrm{C} & -3.14769900 & -0.36374700 & 1.21037000 \\ \mathrm{C} & -3.17598800 & -0.32340600 & -1.19359700 \\ \mathrm{C} & -4.49717800 & -0.02291300 & 1.23369900 \\ \mathrm{C} & -4.52529700 & 0.01780400 & -1.17397000 \\ \mathrm{C} & -5.18913600 & 0.16882600 & 0.04075800 \\ \mathrm{C} & 2.19872600 & 1.01996800 & -0.02394100 \\ \mathrm{C} & 4.01870600 & -0.65835200 & -0.00170900 \\ \mathrm{H} & -0.72926000 & -1.43262900 & 0.85199500 \\ \mathrm{H} & -0.75700200 & -1.42667900 & -0.91614600 \\ \mathrm{H} & -0.43497300 & 1.04149900 & -0.91648400 \\ \mathrm{H} & -0.40169200 & 1.03354300 & 0.86132800 \\ \mathrm{H} & -2.61156300 & -0.51519700 & 2.14193800 \\ \mathrm{H} & -2.66179700 & -0.44365500 & -2.14200700 \\ \mathrm{H} & -5.00833400 & 0.08944100 & 2.18263400 \\ \mathrm{H} & -5.05843900 & 0.16200000 & -2.10635200 \\ \mathrm{H} & -6.24021500 & 0.43146600 & 0.05756500 \\ \mathrm{H} & 2.64963700 & 3.01736700 & 0.06491500 \\ \mathrm{H} & 5.80583000 & -1.58494200 & 0.21233900 \\ \mathrm{H} & 3.70878600 & -2.53892000 & -0.04961900 \\ \mathrm{H} & 4.18716300 & 1.40331000 & -0.07280900 \\ \mathrm{H} & 1.58025900 & -0.85387900 & -0.11275000 \\ \mathrm{H} & 0.97043100 & 2.65028300 & -0.03985000 \\ \mathrm{H} & 5.85696800 & 0.07522100 & 0.54541000\end{array}$

$\mathrm{PFH}+$ - tautomer d

$\begin{array}{lrrr}\mathrm{N} & 1.27521500 & 0.13486100 & -0.08313700 \\ \mathrm{~N} & 3.54488600 & 0.69590400 & 0.10547400 \\ \mathrm{~N} & 1.93182300 & 2.32639000 & 0.15817400 \\ \mathrm{~N} & 5.20692300 & -0.66146300 & -0.68196600 \\ \mathrm{~N} & 3.38621800 & -1.60071900 & 0.55590400 \\ \mathrm{C} & -0.96923200 & -0.83493100 & -0.05885400 \\ \mathrm{C} & -2.44401700 & -0.51661800 & -0.04191800 \\ \mathrm{C} & -0.15293900 & 0.45602100 & -0.05927900 \\ \mathrm{C} & -3.11562700 & -0.33780800 & 1.16868100 \\ \mathrm{C} & -3.14777300 & -0.35111500 & -1.23589500 \\ \mathrm{C} & -4.46616700 & -0.00155100 & 1.18667700 \\ \mathrm{C} & -4.49851300 & -0.01496800 & -1.22135400 \\ \mathrm{C} & -5.16074500 & 0.16068900 & -0.00923700 \\ \mathrm{C} & 2.22950500 & 1.03570800 & 0.05569200 \\ \mathrm{C} & 4.12263000 & -0.59206800 & -0.03130600\end{array}$




$\begin{array}{lrrr}H & -0.69985700 & -1.42743400 & 0.81907900 \\ \mathrm{H} & -0.72003600 & -1.41720300 & -0.94978700 \\ \mathrm{H} & -0.41043600 & 1.05787500 & -0.93595900 \\ \mathrm{H} & -0.37491000 & 1.03292800 & 0.84311600 \\ \mathrm{H} & -2.57737000 & -0.46663300 & 2.10246200 \\ \mathrm{H} & -2.63474400 & -0.49026500 & -2.18226200 \\ \mathrm{H} & -4.97621800 & 0.13015200 & 2.13368500 \\ \mathrm{H} & -5.03373700 & 0.10625300 & -2.15578200 \\ \mathrm{H} & -6.21278700 & 0.41963600 & 0.00351200 \\ \mathrm{H} & 2.64937700 & 3.01243600 & 0.33590600 \\ \mathrm{H} & 5.60147000 & -1.59831600 & -0.63855700 \\ \mathrm{H} & 3.81767800 & -2.51459000 & 0.50976800 \\ \mathrm{H} & 3.02229800 & -1.39411700 & 1.47977700 \\ \mathrm{H} & 4.20222500 & 1.43694700 & -0.10517800 \\ \mathrm{H} & 1.56171100 & -0.83123800 & -0.20296800 \\ \mathrm{H} & 0.98714700 & 2.65860800 & 0.04280800\end{array}$


Table S11. Cartesian coordinates corresponding to the optimized structures of all $\mathrm{PB}$ and $\mathrm{PBH}+$ under study.

$\begin{array}{lrrr}\text { PB - tautomer a } & & \\ \mathrm{C} & -0.94013800 & 0.69443800 & -0.29788400 \\ \mathrm{~N} & -0.39020200 & 1.84846400 & -0.80805400 \\ \mathrm{H} & -1.00085200 & 2.64996800 & -0.83002000 \\ \mathrm{~N} & -2.31312700 & 0.74961900 & -0.28916200 \\ \mathrm{C} & -3.04501900 & -0.26794100 & 0.11009700 \\ \mathrm{~N} & -2.59599900 & -1.47650200 & 0.48324400 \\ \mathrm{H} & -3.22287700 & -2.16757800 & 0.86257500 \\ \mathrm{~N} & -4.39413800 & -0.06664300 & 0.17261200 \\ \mathrm{H} & -4.72861600 & 0.75099900 & -0.31363400 \\ \mathrm{H} & -5.00733300 & -0.86738000 & 0.15660500 \\ \mathrm{~N} & -0.22992100 & -0.33182700 & 0.06865500 \\ \mathrm{C} & 1.17376600 & -0.23984800 & 0.06794800 \\ \mathrm{C} & 1.85431500 & 0.53488500 & 1.01802400 \\ \mathrm{C} & 1.92342900 & -0.98554900 & -0.84948300 \\ \mathrm{C} & 3.24504400 & 0.56951400 & 1.03915000 \\ \mathrm{H} & 1.27962700 & 1.10011200 & 1.74452900 \\ \mathrm{C} & 3.31398300 & -0.94816100 & -0.82414800 \\ \mathrm{H} & 1.39968800 & -1.59362100 & -1.57843800 \\ \mathrm{C} & 3.98297100 & -0.17040600 & 0.11817800 \\ \mathrm{H} & 3.75321300 & 1.17295200 & 1.78272500 \\ \mathrm{H} & 3.87718200 & -1.53113900 & -1.54403400 \\ \mathrm{H} & 5.06574500 & -0.14498600 & 0.13774800 \\ \mathrm{H} & 0.58392000 & 2.03576700 & -0.62750700 \\ \mathrm{H} & -1.58610000 & -1.58845400 & 0.52710200\end{array}$

$\begin{array}{crrr}\text { PB - tautomer b } & & \\ \mathrm{C} & 1.06241600 & 0.81938000 & -0.26259700 \\ \mathrm{~N} & 0.52599500 & 2.02238900 & -0.61302000 \\ \mathrm{H} & 1.18160100 & 2.66762600 & -1.02663500 \\ \mathrm{~N} & 2.37632200 & 0.74571400 & -0.22074200 \\ \mathrm{C} & 3.02134800 & -0.41645800 & 0.13581000 \\ \mathrm{~N} & 2.44175100 & -1.55198500 & 0.38102200 \\ \mathrm{H} & 3.11876500 & -2.27219700 & 0.61604300 \\ \mathrm{~N} & 4.38639500 & -0.22482900 & 0.23894600 \\ \mathrm{H} & 4.97144100 & -1.04430600 & 0.17277000 \\ \mathrm{H} & 4.74149900 & 0.58838600 & -0.24142000 \\ \mathrm{~N} & 0.22366300 & -0.19681100 & 0.03779700\end{array}$




$\begin{array}{lrrc}\mathrm{C} & -1.19112100 & -0.17349400 & 0.04619900 \\ \mathrm{C} & -1.87621900 & -1.23180000 & -0.55466400 \\ \mathrm{C} & -1.90752200 & 0.83832300 & 0.68950000 \\ \mathrm{C} & -3.26506700 & -1.27149100 & -0.52045000 \\ \mathrm{H} & -1.31228500 & -2.01540400 & -1.04740400 \\ \mathrm{C} & -3.29825600 & 0.80089800 & 0.70103300 \\ \mathrm{H} & -1.37757800 & 1.63989200 & 1.18933800 \\ \mathrm{C} & -3.98227500 & -0.25116500 & 0.09908000 \\ \mathrm{H} & -3.78699100 & -2.09797200 & -0.98772300 \\ \mathrm{H} & -3.84641700 & 1.59184400 & 1.19880700 \\ \mathrm{H} & -5.06483900 & -0.27866200 & 0.11725300 \\ \mathrm{H} & 0.73182500 & -1.07762900 & 0.21233600 \\ \mathrm{H} & -0.41573200 & 2.05191800 & -0.97485000\end{array}$

PB - tautomer c

$\begin{array}{lrrr}\mathrm{C} & 1.00304600 & -0.22641500 & 0.41398400 \\ \mathrm{~N} & 0.88198100 & -1.49853900 & 0.57522300 \\ \mathrm{H} & 1.78818800 & -1.89015000 & 0.82058000 \\ \mathrm{~N} & 2.15975300 & 0.54315900 & 0.53030000 \\ \mathrm{C} & 3.23950900 & 0.18648400 & -0.09658600 \\ \mathrm{~N} & 3.38363200 & -0.90476400 & -0.90387400 \\ \mathrm{H} & 4.13244200 & -0.89193300 & -1.58012900 \\ \mathrm{~N} & 4.35108400 & 0.97173800 & 0.02200000 \\ \mathrm{H} & 4.32049100 & 1.64422000 & 0.77340900 \\ \mathrm{H} & 5.25327800 & 0.55152000 & -0.14444200 \\ \mathrm{~N} & -0.08323700 & 0.58275400 & 0.15049900 \\ \mathrm{C} & -1.44465400 & 0.28057500 & 0.04851000 \\ \mathrm{C} & -2.31986900 & 1.36460700 & -0.12945800 \\ \mathrm{C} & -1.97281100 & -1.01521700 & 0.09991800 \\ \mathrm{C} & -3.68537300 & 1.16056000 & -0.24936900 \\ \mathrm{H} & -1.91654700 & 2.37102600 & -0.17201700 \\ \mathrm{C} & -3.34909300 & -1.20192200 & -0.02016000 \\ \mathrm{H} & -1.30912000 & -1.85441400 & 0.23268300 \\ \mathrm{C} & -4.21445400 & -0.12869900 & -0.19436800 \\ \mathrm{H} & -4.33924300 & 2.01405500 & -0.38567900 \\ \mathrm{H} & -3.74286800 & -2.21118300 & 0.02246900 \\ \mathrm{H} & -5.28139500 & -0.28949000 & -0.28711600 \\ \mathrm{H} & 0.16015900 & 1.55880800 & 0.06553500 \\ \mathrm{H} & 2.54431600 & -1.38274600 & -1.19915400\end{array}$




$\begin{array}{lrrr}\text { PB }- \text { tautomer d } & & \\ \mathrm{C} & 1.00004800 & 1.04286800 & -0.03821500 \\ \mathrm{~N} & 0.59793400 & 2.26115000 & -0.04536000 \\ \mathrm{H} & 1.38534000 & 2.90305600 & -0.02369900 \\ \mathrm{~N} & 2.36883900 & 0.70886000 & -0.02377200 \\ \mathrm{C} & 2.99612200 & -0.53077800 & 0.00023200 \\ \mathrm{~N} & 2.33063900 & -1.62434600 & -0.07987700 \\ \mathrm{H} & 2.93541200 & -2.43600800 & -0.00726900 \\ \mathrm{~N} & 4.36745300 & -0.41999500 & 0.05112400 \\ \mathrm{H} & 4.75457400 & 0.38055500 & 0.53119900 \\ \mathrm{H} & 4.86422900 & -1.27695000 & 0.24656400 \\ \mathrm{H} & 2.98095700 & 1.50794800 & -0.10028300 \\ \mathrm{~N} & 0.16498800 & -0.03507600 & -0.04168400 \\ \mathrm{C} & -1.23396000 & -0.06756300 & -0.01411700 \\ \mathrm{C} & -1.81776700 & -1.34545300 & -0.01865800 \\ \mathrm{C} & -2.06683300 & 1.05715300 & 0.01844700 \\ \mathrm{C} & -3.19481900 & -1.49636600 & 0.00929900 \\ \mathrm{H} & -1.17570200 & -2.21981300 & -0.04341000 \\ \mathrm{C} & -3.45071600 & 0.88714900 & 0.04698700 \\ \mathrm{H} & -1.63149200 & 2.04322900 & 0.01843000 \\ \mathrm{C} & -4.02667900 & -0.37714900 & 0.04285500 \\ \mathrm{H} & -3.61993600 & -2.49325100 & 0.00527500 \\ \mathrm{H} & -4.08240100 & 1.76791600 & 0.07201300 \\ \mathrm{H} & -5.10325000 & -0.49293200 & 0.06485800 \\ \mathrm{H} & 0.65092300 & -0.93708200 & -0.06767000 \\ & & & \end{array}$

$\mathrm{PBH}+$ - tautomer a

$\begin{array}{lrrr}\mathrm{C} & 0.99715200 & 0.76291100 & 0.34114300 \\ \mathrm{~N} & 0.43539200 & 1.95123200 & 0.55793300 \\ \mathrm{~N} & 2.32157400 & 0.68884700 & 0.44348500 \\ \mathrm{C} & 3.05792200 & -0.25464800 & -0.13776300 \\ \mathrm{~N} & 2.67551100 & -0.99106400 & -1.19573100 \\ \mathrm{H} & 3.33368100 & -1.60389700 & -1.65376700 \\ \mathrm{~N} & 4.27818300 & -0.45829900 & 0.36036500 \\ \mathrm{~N} & 0.19867200 & -0.29807400 & 0.08270600 \\ \mathrm{C} & -1.22823900 & -0.25303600 & 0.01988100 \\ \mathrm{C} & -1.85725500 & 0.52615300 & -0.94888600 \\ \mathrm{C} & -1.97400800 & -1.01835200 & 0.91224100 \\ \mathrm{C} & -3.24644500 & 0.55465100 & -1.00532400 \\ \mathrm{H} & -1.26163100 & 1.09494600 & -1.65364600 \\ \mathrm{C} & -3.36238800 & -1.00075800 & 0.83274700\end{array}$




$\begin{array}{lrrr}\mathrm{H} & -1.46710600 & -1.61880600 & 1.65854000 \\ \mathrm{C} & -4.00009600 & -0.20981100 & -0.11879800 \\ \mathrm{H} & -3.73760800 & 1.16353600 & -1.75424800 \\ \mathrm{H} & -3.94450200 & -1.59945100 & 1.52242900 \\ \mathrm{H} & -5.08165800 & -0.19205400 & -0.17208100 \\ \mathrm{H} & 0.62259800 & -1.21254000 & 0.17326100 \\ \mathrm{H} & 4.94763000 & -1.06946200 & -0.07912600 \\ \mathrm{H} & -0.56150300 & 2.06492400 & 0.65948800 \\ \mathrm{H} & 4.55548200 & 0.06415300 & 1.17612300 \\ \mathrm{H} & 1.86668600 & -0.71659800 & -1.73257700 \\ \mathrm{H} & 1.04274900 & 2.73409000 & 0.74285100\end{array}$

$\mathrm{PBH}+$ - tautomer b

$\begin{array}{lrrr}\mathrm{C} & 0.87117500 & 0.72554900 & -0.28643000 \\ \mathrm{~N} & 0.39448700 & 1.93702800 & -0.67510700 \\ \mathrm{~N} & 2.27837300 & 0.67844200 & -0.27158600 \\ \mathrm{C} & 3.06207300 & -0.35683500 & 0.12689400 \\ \mathrm{~N} & 2.52814900 & -1.49386000 & 0.51594300 \\ \mathrm{H} & 3.10779300 & -2.25002200 & 0.84702300 \\ \mathrm{~N} & 4.37804100 & -0.17588800 & 0.10588000 \\ \mathrm{~N} & 0.19667700 & -0.32593500 & -0.01745900 \\ \mathrm{C} & -1.21592900 & -0.24437800 & 0.02717900 \\ \mathrm{C} & -1.97129500 & -0.92516700 & -0.92940400 \\ \mathrm{C} & -1.86428700 & 0.45878300 & 1.04698200 \\ \mathrm{C} & -3.36073400 & -0.89062900 & -0.87248300 \\ \mathrm{H} & -1.45951700 & -1.47664800 & -1.70935000 \\ \mathrm{C} & -3.25435700 & 0.48734500 & 1.09789600 \\ \mathrm{H} & -1.27346300 & 0.97027900 & 1.79922900 \\ \mathrm{C} & -4.00751100 & -0.18488100 & 0.13898600 \\ \mathrm{H} & -3.93891200 & -1.41987400 & -1.62073700 \\ \mathrm{H} & -3.74815900 & 1.03305900 & 1.89324200 \\ \mathrm{H} & -5.08953900 & -0.16278500 & 0.18217000 \\ \mathrm{H} & 5.00568700 & -0.92417300 & 0.35888500 \\ \mathrm{H} & -0.60249600 & 2.07936300 & -0.60098900 \\ \mathrm{H} & 4.78787800 & 0.71200200 & -0.14003600 \\ \mathrm{H} & 1.50982800 & -1.57145800 & 0.46849200 \\ \mathrm{H} & 0.95743200 & 2.76370700 & -0.54108700 \\ \mathrm{H} & 2.75856700 & 1.48930800 & -0.63825000\end{array}$


$\mathrm{PBH}+$ - tautomer c

$\begin{array}{lrrr}\mathrm{C} & -0.95877900 & 0.77138800 & -0.32038300 \\ \mathrm{~N} & -0.44116700 & 1.91894200 & -0.74331800 \\ \mathrm{H} & -1.02196700 & 2.71030900 & -0.97340400 \\ \mathrm{~N} & -2.31351600 & 0.66499800 & -0.27028000 \\ \mathrm{C} & -3.05556100 & -0.45251500 & 0.16112400 \\ \mathrm{~N} & -2.45374100 & -1.54253600 & 0.44095100 \\ \mathrm{H} & -3.08896000 & -2.25894200 & 0.77794800 \\ \mathrm{~N} & -4.39639500 & -0.20843900 & 0.15789400 \\ \mathrm{H} & -2.83985400 & 1.44078400 & -0.64973100 \\ \mathrm{~N} & -0.19921800 & -0.24775800 & 0.03893000 \\ \mathrm{C} & 1.23048900 & -0.21304800 & 0.06427700 \\ \mathrm{C} & 1.89331500 & 0.65253900 & 0.93102500 \\ \mathrm{C} & 1.93796600 & -1.07926200 & -0.76360000 \\ \mathrm{C} & 3.28402000 & 0.65963000 & 0.95263000 \\ \mathrm{H} & 1.32426900 & 1.30287700 & 1.58557300 \\ \mathrm{C} & 3.32798500 & -1.07632700 & -0.72166500 \\ \mathrm{H} & 1.39930700 & -1.74359700 & -1.42843200 \\ \mathrm{C} & 4.00108900 & -0.20480700 & 0.13010600 \\ \mathrm{H} & 3.80433400 & 1.33273800 & 1.62260700 \\ \mathrm{H} & 3.88365600 & -1.75159300 & -1.36043300 \\ \mathrm{H} & 5.08385200 & -0.20136800 & 0.15528100 \\ \mathrm{H} & -0.73098800 & -1.10469800 & 0.27073300 \\ \mathrm{H} & -4.98407600 & -0.92185800 & 0.56352500 \\ \mathrm{H} & 0.55827200 & 2.01815600 & -0.84498000 \\ \mathrm{H} & -4.72272600 & 0.73515000 & 0.31099100\end{array}$

$\mathrm{PBH}+$ - tautomer d

$\begin{array}{lrrr}\mathrm{C} & 0.92766700 & 0.85509300 & -0.40257200 \\ \mathrm{~N} & 0.58662900 & 2.07180300 & -0.38222100 \\ \mathrm{~N} & 2.31583200 & 0.57467400 & -0.52937000 \\ \mathrm{C} & 3.04471000 & -0.33263800 & 0.16296700 \\ \mathrm{~N} & 2.48373000 & -1.18564400 & 1.00250600 \\ \mathrm{H} & 3.04141800 & -1.88407800 & 1.47112400 \\ \mathrm{~N} & 4.35737500 & -0.33892900 & -0.02305400 \\ \mathrm{H} & 2.83096900 & 1.34785800 & -0.93305100 \\ \mathrm{~N} & 0.15175700 & -0.29075700 & -0.33301000 \\ \mathrm{C} & -1.25620600 & -0.22174900 & -0.14002300 \\ \mathrm{C} & -2.10851700 & -0.86086100 & -1.03708000 \\ \mathrm{C} & -1.77397400 & 0.44805400 & 0.96846600 \\ \mathrm{C} & -3.48254700 & -0.83129000 & -0.82094900\end{array}$




$\begin{array}{lrrr}\mathrm{H} & -1.69739400 & -1.37792700 & -1.89685200 \\ \mathrm{C} & -3.14936200 & 0.48974000 & 1.16374600 \\ \mathrm{H} & -1.10225600 & 0.92115200 & 1.67565600 \\ \mathrm{C} & -4.00758200 & -0.15085000 & 0.27320800 \\ \mathrm{H} & -4.14207700 & -1.33457100 & -1.51739800 \\ \mathrm{H} & -3.54887200 & 1.01407700 & 2.02325800 \\ \mathrm{H} & -5.07817900 & -0.12211900 & 0.43367700 \\ \mathrm{H} & 0.46133500 & -1.03882900 & -0.94474200 \\ \mathrm{H} & 4.94984200 & -0.97969300 & 0.48257400 \\ \mathrm{H} & -0.42689600 & 2.17126000 & -0.40341400 \\ \mathrm{H} & 4.79337700 & 0.26154300 & -0.70593800 \\ \mathrm{H} & 1.48633900 & -1.16970400 & 1.16457300\end{array}$


Table S12. Cartesian coordinates corresponding to the optimized structures of $\left\{(\mathrm{MF}) \cdot\left(\mathrm{H}_{2} \mathrm{O}\right)_{20}\right\}$, $\left\{(\mathrm{MFH}) \cdot\left(\mathrm{H}_{2} \mathrm{O}\right)_{20}\right\}^{+}$and $\left\{\left(\mathrm{MFH}_{2}\right) \cdot\left(\mathrm{H}_{2} \mathrm{O}\right)_{20}\right\}^{2+}$ under study.

$\left\{(\mathrm{MF}) \cdot\left(\mathrm{H}_{2} \mathrm{O}\right)_{20}\right\}$

\begin{tabular}{|c|c|c|c|}
\hline $\mathrm{N}$ & -2.52353500 & -2.77475100 & 1.17311400 \\
\hline $\mathrm{N}$ & -2.91280000 & -1.53874700 & -0.82484600 \\
\hline $\mathrm{N}$ & 0.81498400 & 0.04812400 & -0.10706400 \\
\hline $\mathrm{N}$ & -1.26077000 & 0.71780100 & -0.80760700 \\
\hline $\mathrm{C}$ & -2.17349100 & -2.51067100 & 2.57492100 \\
\hline $\mathrm{C}$ & -3.80744900 & -3.44755400 & 1.01472500 \\
\hline $\mathrm{C}$ & -2.17920200 & -1.80051800 & 0.22531500 \\
\hline $\mathrm{C}$ & -0.50160600 & -0.19153100 & -0.18537400 \\
\hline $\mathrm{H}$ & -1.12891200 & -2.21900500 & 2.65024000 \\
\hline $\mathrm{H}$ & -2.32102400 & -3.42802000 & 3.14405400 \\
\hline $\mathrm{H}$ & -2.80581500 & -1.72386800 & 3.00274300 \\
\hline $\mathrm{H}$ & -3.87218200 & -4.24062600 & 1.75744800 \\
\hline $\mathrm{H}$ & -3.87797800 & -3.90878500 & 0.02946700 \\
\hline $\mathrm{H}$ & -4.65422900 & -2.76498100 & 1.15986000 \\
\hline $\mathrm{H}$ & -3.76919400 & -2.08195500 & -0.83191600 \\
\hline $\mathrm{H}$ & 1.41364200 & -0.69567300 & 0.24149300 \\
\hline $\mathrm{H}$ & -2.25451800 & 0.57348300 & -0.88789200 \\
\hline $\mathrm{H}$ & -0.82530300 & 1.43250400 & -1.36867900 \\
\hline $\mathrm{H}$ & 1.23750900 & 0.83194600 & -0.58157600 \\
\hline $\mathrm{O}$ & 2.15796700 & -2.22839700 & 1.24512800 \\
\hline $\mathrm{H}$ & 2.32171200 & -1.67071100 & 2.02500300 \\
\hline $\mathrm{H}$ & 1.34260500 & -2.74076300 & 1.37280000 \\
\hline $\mathrm{O}$ & 3.70193300 & -3.30349800 & -0.73758200 \\
\hline $\mathrm{H}$ & 3.23506900 & -2.97983500 & 0.05833700 \\
\hline $\mathrm{H}$ & 2.98584000 & -3.41310800 & -1.37897400 \\
\hline $\mathrm{O}$ & 2.96769800 & 3.31838700 & -0.15703500 \\
\hline $\mathrm{H}$ & 3.30128900 & 2.40343900 & 0.00249200 \\
\hline $\mathrm{H}$ & 3.72103300 & 3.83560600 & -0.45928300 \\
\hline $\mathrm{O}$ & 0.49654300 & 3.04157200 & -1.43617800 \\
\hline $\mathrm{H}$ & 1.42788200 & 3.27411800 & -1.27460100 \\
\hline $\mathrm{H}$ & 0.08701400 & 3.11250100 & -0.54580400 \\
\hline $\mathrm{O}$ & -3.80309700 & 0.92715100 & 1.20192800 \\
\hline $\mathrm{H}$ & -3.70233500 & 1.74411900 & 0.66965700 \\
\hline $\mathrm{H}$ & -3.92665000 & 0.21929700 & 0.55726000 \\
\hline $\mathrm{O}$ & -0.97545100 & -1.51056800 & -2.98234300 \\
\hline $\mathrm{H}$ & -1.70639600 & -1.45644700 & -2.33547200 \\
\hline $\mathrm{H}$ & -0.61805500 & -0.60967200 & -3.07228000 \\
\hline $\mathrm{N}$ & -0.95449300 & -1.25649600 & 0.47333300 \\
\hline $\mathrm{O}$ & -1.33688200 & 0.84997200 & 2.50394300 \\
\hline $\mathrm{H}$ & -2.26707100 & 0.88699400 & 2.19866700 \\
\hline
\end{tabular}




$\begin{array}{lrrr}\mathrm{H} & -1.01438600 & 0.01602800 & 2.12592400 \\ \mathrm{O} & 2.61007000 & -0.81898300 & -2.71553300 \\ \mathrm{H} & 2.16124100 & -1.48814100 & -2.17016600 \\ \mathrm{H} & 3.48910800 & -0.69873300 & -2.32592300 \\ \mathrm{O} & 5.01289500 & -1.00394700 & -1.23497700 \\ \mathrm{H} & 4.72649300 & -1.93074100 & -1.07727700 \\ \mathrm{H} & 5.94550100 & -1.01727900 & -1.46693400 \\ \mathrm{O} & 1.03106500 & -2.92394300 & -1.70643900 \\ \mathrm{H} & 0.67927800 & -3.19495200 & -0.84203800 \\ \mathrm{H} & 0.28286100 & -2.50039600 & -2.17610200 \\ \mathrm{O} & 0.09397700 & -4.04691600 & 0.77522400 \\ \mathrm{H} & -0.84172300 & -3.79269900 & 0.87198100 \\ \mathrm{H} & 0.13499500 & -5.00108700 & 0.89097900 \\ \mathrm{O} & -3.96918300 & 0.96062600 & -2.08700500 \\ \mathrm{H} & -3.69914200 & 0.03189200 & -2.10180300 \\ \mathrm{H} & -3.30078100 & 1.44552700 & -2.58952900 \\ \mathrm{O} & -2.01943900 & 3.08058200 & -2.67018700 \\ \mathrm{H} & -2.31291300 & 3.62926500 & -3.40496400 \\ \mathrm{H} & -1.09872700 & 3.33845300 & -2.49709800 \\ \mathrm{O} & -0.63664100 & 3.16888100 & 1.04884300 \\ \mathrm{H} & -0.78014700 & 2.30746300 & 1.48541100 \\ \mathrm{H} & 0.03050200 & 3.64268000 & 1.57623300 \\ \mathrm{O} & -3.21040100 & 3.32607300 & -0.04027000 \\ \mathrm{H} & -2.99106200 & 3.24350400 & -0.98117600 \\ \mathrm{H} & -2.35484700 & 3.49441400 & 0.38809300 \\ \mathrm{O} & 1.71319100 & 4.00957600 & 2.20682400 \\ \mathrm{H} & 2.00738900 & 4.83186400 & 2.60989400 \\ \mathrm{H} & 2.26150600 & 3.86571700 & 1.40826700 \\ \mathrm{H} & 3.62821400 & 0.79012200 & 0.37532900 \\ \mathrm{O} & 4.20022000 & 0.17165600 & -0.11096700 \\ \mathrm{H} & 3.48043200 & 0.42726500 & 1.26565400 \\ \mathrm{H} & 1.09999600 & 1.68208800 & 3.68653200 \\ \mathrm{H} & 0.17869900 & 1.49060100 & 3.45213800 \\ \mathrm{H} & 1.33178300 & 2.51681200 & 3.24474300 \\ \mathrm{H} & 2.88987500 & -0.17993600 & 2.92563400 \\ \mathrm{H} & 3.53638900 & -0.27880000 & 3.63108100 \\ \mathrm{H} & & 0.84691300 & -3.40435300 \\ \mathrm{H} & & & \\ \mathrm{H} & & & \end{array}$

$\left\{(\mathrm{MFH}) \cdot\left(\mathrm{H}_{2} \mathrm{O}\right)_{20}\right\}^{+}$

$\begin{array}{lrrr}\mathrm{N} & -3.21910700 & -0.00983100 & -0.85012600 \\ \mathrm{~N} & -1.65351600 & 1.12949500 & -2.08717000 \\ \mathrm{~N} & 1.08554800 & -0.79267500 & 0.38927500\end{array}$ 


$\begin{array}{lrrr}\mathrm{N} & 0.41008500 & 1.36014600 & -0.00308500 \\ \mathrm{C} & -3.61913000 & -0.79921400 & 0.30939200 \\ \mathrm{C} & -4.28841600 & 0.53491600 & -1.67869400 \\ \mathrm{C} & -1.94689400 & 0.23764400 & -1.13672200 \\ \mathrm{C} & 0.14667100 & 0.04324000 & -0.09114100 \\ \mathrm{H} & -2.77844600 & -0.92593700 & 0.98638900 \\ \mathrm{H} & -3.98582500 & -1.77927700 & -0.00427000 \\ \mathrm{H} & -4.41769500 & -0.27100000 & 0.83322500 \\ \mathrm{H} & -5.18899100 & -0.04805900 & -1.49813400 \\ \mathrm{H} & -4.02192300 & 0.44974800 & -2.73258600 \\ \mathrm{H} & -4.49063100 & 1.58141100 & -1.43170500 \\ \mathrm{H} & -2.31413100 & 1.85977800 & -2.31926100 \\ \mathrm{H} & 1.03642700 & -1.78479700 & 0.16769200 \\ \mathrm{H} & -0.34497600 & 2.02194400 & -0.11388000 \\ \mathrm{H} & 1.20996600 & 1.63788100 & 0.55983600 \\ \mathrm{H} & -0.68995900 & 1.26957100 & -2.39148100 \\ \mathrm{H} & 1.95841500 & -0.39934500 & 0.71581900 \\ \mathrm{O} & -1.51595600 & -3.23879700 & -0.37763500 \\ \mathrm{H} & -1.34271100 & -2.27589400 & -0.29469500 \\ \mathrm{H} & -2.12313500 & -3.46849100 & 0.35408000 \\ \mathrm{O} & 1.18984600 & -3.72404800 & -0.18994300 \\ \mathrm{H} & 0.21858800 & -3.78616900 & -0.25753100 \\ \mathrm{H} & 1.55318200 & -3.57206000 & -1.08887200 \\ \mathrm{O} & 4.48617200 & 1.29299900 & -0.38634800 \\ \mathrm{H} & 4.46276200 & 0.33188100 & -0.57263000 \\ \mathrm{H} & 3.26960700 & -2.97958900 & 1.32571500 \\ \mathrm{H} & -438600 & 1.56422300 & -0.46852400 \\ \mathrm{O} & 2.64711000 & 2.95535500 & -1.60266000 \\ \mathrm{H} & 3.16511800 & 3.59279400 & -2.10684500 \\ \mathrm{H} & 3.30192000 & 2.37609700 & -1.16024300 \\ \mathrm{O} & -2.28382600 & 1.93662100 & 1.31893200 \\ \mathrm{H} & -1.80880600 & 2.72556500 & 1.68603100 \\ \mathrm{H} & -3.15955700 & 1.92530400 & 1.72144600 \\ \mathrm{O} & 0.95783700 & 1.46299400 & -3.19873400 \\ \mathrm{H} & 1.59592100 & 1.94804500 & -2.63880200 \\ \mathrm{H} & 0.97633600 & 1.89398900 & -4.05951400 \\ \mathrm{H} & -0.98381000 & -0.49464400 & -0.52179000 \\ \mathrm{H} & -32394800 & 0.03369400 & 2.80620400 \\ \mathrm{H} & & -3.70262200 & 1.61128500\end{array}$




\begin{tabular}{|c|c|c|c|}
\hline $\mathrm{H}$ & 2.47891100 & -3.39364100 & 0.91581700 \\
\hline $\mathrm{O}$ & 0.50054400 & -1.30840900 & -3.53148300 \\
\hline $\mathrm{H}$ & -0.41523000 & -1.52013400 & -3.27389900 \\
\hline $\mathrm{H}$ & 0.64282400 & -0.36092600 & -3.38345500 \\
\hline $\mathrm{O}$ & -2.08063700 & -2.20698800 & -2.90666800 \\
\hline $\mathrm{H}$ & -2.04413600 & -2.77241500 & -2.11525200 \\
\hline $\mathrm{H}$ & -2.49140500 & -2.73654700 & -3.59614500 \\
\hline $\mathrm{O}$ & -2.54134000 & 3.72943800 & -1.03840000 \\
\hline $\mathrm{H}$ & -2.63406000 & 3.15317500 & -0.26680900 \\
\hline $\mathrm{H}$ & -1.60027700 & 3.95830000 & -1.05290100 \\
\hline $\mathrm{O}$ & 0.33164700 & 4.24276400 & -0.62871500 \\
\hline $\mathrm{H}$ & 0.47668100 & 5.16997700 & -0.84762200 \\
\hline $\mathrm{H}$ & 1.13792300 & 3.77991500 & -0.92780600 \\
\hline $\mathrm{O}$ & 0.86210200 & 2.28674900 & 3.53375600 \\
\hline $\mathrm{H}$ & 0.33402200 & 1.47368100 & 3.48245900 \\
\hline $\mathrm{H}$ & 1.65180600 & 2.09707700 & 3.00110000 \\
\hline $\mathrm{O}$ & -0.69767800 & 3.93880400 & 2.00470400 \\
\hline $\mathrm{H}$ & -0.21003800 & 4.16409600 & 1.19619600 \\
\hline $\mathrm{H}$ & -0.06365900 & 3.48142200 & 2.59401000 \\
\hline $\mathrm{O}$ & 2.78740400 & 1.25829000 & 1.78694800 \\
\hline $\mathrm{H}$ & 2.99318300 & 0.49590800 & 2.36780200 \\
\hline $\mathrm{H}$ & 3.56982900 & 1.42565500 & 1.23586300 \\
\hline $\mathrm{O}$ & 3.01234600 & -1.00818700 & 3.25570600 \\
\hline $\mathrm{H}$ & 3.03811000 & -1.79112100 & 2.67593200 \\
\hline $\mathrm{H}$ & 2.32882700 & -1.17510000 & 3.91115000 \\
\hline $\mathrm{O}$ & -2.80423800 & -1.75696600 & 3.66178500 \\
\hline $\mathrm{H}$ & -2.11186100 & -1.10148600 & 3.46048000 \\
\hline $\mathrm{H}$ & -3.55465000 & -1.25673800 & 3.99507100 \\
\hline $\mathrm{O}$ & -3.21925100 & -3.69068900 & 1.74615700 \\
\hline $\mathrm{H}$ & -3.09808000 & -3.03063900 & 2.45734200 \\
\hline $\mathrm{H}$ & -3.21473100 & -4.54908000 & 2.17894300 \\
\hline $\mathrm{O}$ & 4.33853400 & -1.45958800 & -0.76628400 \\
\hline $\mathrm{H}$ & 5.21023300 & -1.78958900 & -1.01163100 \\
\hline $\mathrm{H}$ & 4.07481700 & -1.97749700 & 0.02031700 \\
\hline $\multicolumn{4}{|c|}{\left(\mathrm{MFH}_{2}\right) \cdot\left(\mathrm{H}_{2} \mathrm{O}\right)_{20}}^{2+}$ \\
\hline $\mathrm{N}$ & -2.69568000 & -1.91538700 & -0.90605900 \\
\hline $\mathrm{N}$ & -2.29344300 & 0.17492900 & -1.82739700 \\
\hline $\mathrm{N}$ & 1.39580200 & -0.07436800 & -0.01775500 \\
\hline $\mathrm{N}$ & -0.50274200 & 1.19952100 & 0.25278500 \\
\hline $\mathrm{C}$ & -2.35385700 & -2.94438500 & 0.07686300 \\
\hline $\mathrm{C}$ & -3.99103600 & -2.02243800 & -1.57287000 \\
\hline $\mathrm{C}$ & -1.91373100 & -0.88998000 & -1.14402300 \\
\hline $\mathrm{C}$ & 0.08684500 & 0.09581300 & -0.16123300 \\
\hline
\end{tabular}




\begin{tabular}{|c|c|c|c|}
\hline $\mathrm{H}$ & -1.67363900 & -2.54003300 & 0.82401100 \\
\hline $\mathrm{H}$ & -1.89948500 & -3.80558300 & -0.41704700 \\
\hline $\mathrm{H}$ & -3.27220300 & -3.25560600 & 0.57325900 \\
\hline $\mathrm{H}$ & -4.24937800 & -3.07685700 & -1.64291800 \\
\hline $\mathrm{H}$ & -3.92373100 & -1.60462700 & -2.57642800 \\
\hline $\mathrm{H}$ & -4.76100900 & -1.49805800 & -1.00172000 \\
\hline $\mathrm{H}$ & -3.25677500 & 0.50493700 & -1.85546800 \\
\hline $\mathrm{H}$ & 1.86073400 & -0.76058800 & -0.60707400 \\
\hline $\mathrm{H}$ & -1.51190200 & 1.26619800 & 0.31362900 \\
\hline $\mathrm{H}$ & 0.06503500 & 1.89449600 & 0.75290400 \\
\hline $\mathrm{H}$ & -1.56178800 & 0.77958600 & -2.20655900 \\
\hline $\mathrm{H}$ & 1.94706100 & 0.66831000 & 0.39095100 \\
\hline $\mathrm{O}$ & 0.94759600 & -3.24698700 & -0.49827700 \\
\hline $\mathrm{H}$ & 0.65740000 & -3.69341000 & 0.33626100 \\
\hline $\mathrm{H}$ & 0.87380900 & -3.90794700 & -1.19549900 \\
\hline $\mathrm{O}$ & 3.63870100 & -2.47436400 & -0.15756500 \\
\hline $\mathrm{H}$ & 2.75373200 & -2.83814800 & -0.33102000 \\
\hline $\mathrm{H}$ & 3.96330600 & -2.07337000 & -0.98823700 \\
\hline $\mathrm{O}$ & 2.98396100 & 3.53931800 & -0.06820900 \\
\hline $\mathrm{H}$ & 3.60430900 & 2.80022000 & -0.23754200 \\
\hline $\mathrm{H}$ & 3.51500900 & 4.34241600 & -0.04588500 \\
\hline $\mathrm{O}$ & 0.74487700 & 3.64491600 & -1.68954900 \\
\hline $\mathrm{H}$ & 0.92888800 & 4.33868700 & -2.33336500 \\
\hline $\mathrm{H}$ & 1.53024500 & 3.62054000 & -1.10416700 \\
\hline $\mathrm{O}$ & -2.96340100 & -0.04636400 & 1.39133300 \\
\hline $\mathrm{H}$ & -3.27281100 & 0.87864300 & 1.37326400 \\
\hline $\mathrm{H}$ & -3.71325000 & -0.58619800 & 1.66127400 \\
\hline $\mathrm{O}$ & 0.11166600 & 1.27728400 & -2.91956600 \\
\hline $\mathrm{H}$ & 0.42107400 & 2.11206300 & -2.50982300 \\
\hline $\mathrm{H}$ & -0.00871100 & 1.47731200 & -3.85491400 \\
\hline $\mathrm{N}$ & -0.60265300 & -0.94474700 & -0.70409700 \\
\hline $\mathrm{O}$ & -0.90684500 & -0.19006100 & 3.27692100 \\
\hline $\mathrm{H}$ & -1.49661400 & -0.14352600 & 2.50198000 \\
\hline $\mathrm{H}$ & 0.01906800 & -0.13972900 & 2.98204500 \\
\hline $\mathrm{O}$ & 4.37245800 & -0.89203200 & -2.29454100 \\
\hline $\mathrm{H}$ & 3.47807800 & -0.89606700 & -2.67690700 \\
\hline $\mathrm{H}$ & 4.45033300 & -0.04864900 & -1.82227600 \\
\hline $\mathrm{O}$ & 3.97938000 & -0.54191400 & 1.59386900 \\
\hline $\mathrm{H}$ & 4.72903300 & -0.73418000 & 2.16838000 \\
\hline $\mathrm{H}$ & 3.84703900 & -1.34567700 & 1.02464200 \\
\hline $\mathrm{O}$ & 1.66195500 & -1.08201400 & -2.7270160 \\
\hline
\end{tabular}




$\begin{array}{lrrr}\mathrm{H} & 1.04019700 & -1.78431100 & -2.97617500 \\ \mathrm{H} & 1.17387700 & -0.25066000 & -2.86520000 \\ \mathrm{O} & -0.63970200 & -2.67044200 & -3.15175000 \\ \mathrm{H} & -0.77011600 & -3.61112800 & -3.31274600 \\ \mathrm{H} & -1.13506500 & -2.23382900 & -3.85402400 \\ \mathrm{O} & -4.25806800 & 2.18874700 & -1.50794000 \\ \mathrm{H} & -4.59691300 & 2.22417800 & -0.60689600 \\ \mathrm{H} & -3.51750300 & 2.81646700 & -1.50927500 \\ \mathrm{O} & -1.99334100 & 3.91650300 & -1.03245100 \\ \mathrm{H} & -2.16765000 & 4.85072600 & -1.18903500 \\ \mathrm{H} & -1.03961000 & 3.79665700 & -1.19985000 \\ \mathrm{O} & -1.18899000 & 2.71140500 & 3.36370600 \\ \mathrm{H} & -1.11311100 & 1.76943300 & 3.58577900 \\ \mathrm{H} & -0.33337000 & 2.94136600 & 2.96947100 \\ \mathrm{O} & -2.96943500 & 2.65830600 & 1.25116200 \\ \mathrm{H} & -2.63349700 & 3.25268200 & 0.55853000 \\ \mathrm{H} & -2.42170900 & 2.82239700 & 2.04472500 \\ \mathrm{O} & 1.24991600 & 2.64676700 & 1.88236500 \\ \mathrm{H} & 1.66545500 & 1.95892300 & 2.43461600 \\ \mathrm{H} & 1.95321400 & 3.12587100 & 1.41026600 \\ \mathrm{O} & 1.85137300 & 0.27244200 & 3.10869500 \\ \mathrm{H} & 2.59898800 & -0.13701500 & 2.62506100 \\ \mathrm{H} & 2.08174100 & 0.22895800 & 4.04318400 \\ \mathrm{O} & -1.25783800 & -2.93331700 & 3.61200100 \\ \mathrm{H} & -1.16304200 & -1.96199000 & 3.61003400 \\ \mathrm{H} & -2.18570000 & -3.11479100 & 3.78640200 \\ \mathrm{O} & 0.08004500 & -4.39608200 & 1.74641300 \\ \mathrm{H} & -0.38991600 & -3.88037000 & 2.43473300 \\ \mathrm{H} & 0.68416200 & -4.98100200 & 2.21273600 \\ \mathrm{H} & 4.58583600 & 1.30268100 & -0.39012900 \\ & 5.51155400 & 1.57088800 & -0.40822100 \\ \mathrm{H} & -0.49408600 & 0.71655200 & 0.38850500 \\ \mathrm{H} & & -1.84721600 & -0.76889300\end{array}$


Table S13. Cartesian coordinates corresponding to the optimized structures of $\left\{(\mathrm{PF}) \cdot\left(\mathrm{H}_{2} \mathrm{O}\right)_{20}\right\}$, $\left\{(\mathrm{PFH}) \cdot\left(\mathrm{H}_{2} \mathrm{O}\right)_{20}\right\}^{+}$and $\left\{\left(\mathrm{PFH}_{2}\right) \cdot\left(\mathrm{H}_{2} \mathrm{O}\right)_{20}\right\}^{2+}$ under study.

$\left\{(\mathrm{PF}) \cdot\left(\mathrm{H}_{2} \mathrm{O}\right)_{20}\right\}$

\begin{tabular}{|c|c|c|c|}
\hline & -1.00384200 & 0.32840200 & 0.41730000 \\
\hline & 1.27788100 & -0.16993900 & -0.02211400 \\
\hline & -0.31415700 & -1.78519600 & -0.35857500 \\
\hline & 3.04677200 & 1.28738500 & -0.03051200 \\
\hline & 0.93006900 & 2.17307000 & -0.14815400 \\
\hline & -3.36388000 & 0.93924200 & 0.69879300 \\
\hline & -4.77781900 & 0.42477900 & 0.61708400 \\
\hline & -2.36851900 & -0.17710800 & 0.38456300 \\
\hline & -5.48037800 & 0.48183400 & -0.58892000 \\
\hline & -5.38908800 & -0.17445200 & 1.72078000 \\
\hline & -6.76560800 & -0.04372800 & -0.69022200 \\
\hline & -6.67417100 & -0.70147000 & 1.62432500 \\
\hline $\mathrm{C}$ & -7.36620000 & -0.63759800 & 0.41730600 \\
\hline & -0.06563300 & -0.48075000 & 0.04756000 \\
\hline 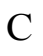 & 1.70036200 & 1.08979800 & -0.06341300 \\
\hline $\mathrm{H}$ & -3.21285600 & 1.74239300 & -0.02507400 \\
\hline H & -3.16227500 & 1.33368900 & 1.69917400 \\
\hline 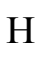 & -2.50584900 & -0.99287200 & 1.10884700 \\
\hline$t$ & -2.60413300 & -0.59053800 & -0.60671700 \\
\hline$\theta$ & -5.01169300 & 0.94657100 & -1.45118700 \\
\hline $\mathrm{H}$ & -4.85305100 & -0.22435800 & 2.66370500 \\
\hline & -7.29917700 & 0.01258500 & -1.63206900 \\
\hline $\mathrm{H}$ & -7.13613700 & -1.15941200 & 2.49137500 \\
\hline H & -8.36744800 & -1.04498500 & 0.34130900 \\
\hline 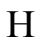 & 0.51076000 & -2.37699200 & -0.37691300 \\
\hline $\mathrm{H}$ & 3.62639400 & 0.46940200 & -0.24526300 \\
\hline $\mathrm{H}$ & -1.14098200 & -2.24384600 & 0.01529000 \\
\hline $\mathrm{H}$ & 3.37515000 & 2.19009500 & -0.36001600 \\
\hline $\mathrm{H}$ & 1.35558100 & 3.08660300 & -0.14728200 \\
\hline $\mathrm{H}$ & -0.07694700 & 2.09270000 & -0.07725400 \\
\hline $\mathrm{O}$ & -1.42273600 & 3.92041100 & -0.15241900 \\
\hline $\mathrm{H}$ & -2.09144400 & 4.02605000 & 0.52988800 \\
\hline $\mathrm{H}$ & -1.85660900 & 3.48242300 & -0.91790400 \\
\hline $\mathrm{O}$ & 0.93727200 & 5.21333500 & -0.08948700 \\
\hline $\mathrm{H}$ & 0.01208800 & 4.90158300 & -0.19482600 \\
\hline $\mathrm{H}$ & 0.90456600 & 6.17456800 & -0.05653300 \\
\hline $\mathrm{O}$ & 4.67922900 & -1.02912000 & -0.38306300 \\
\hline $\mathrm{H}$ & 4.99636700 & -1.26730300 & 0.50248100 \\
\hline$\pi$ & 4.45620700 & -1.87732300 & -0.80977800 \\
\hline 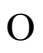 & 3.08562900 & 3.97252000 & -1.38718700 \\
\hline
\end{tabular}




\begin{tabular}{|c|c|c|c|}
\hline $\mathrm{H}$ & 3.88202400 & 4.48286400 & -1.56383500 \\
\hline $\mathrm{H}$ & 2.41202300 & 4.60744200 & -1.08526000 \\
\hline $\mathrm{O}$ & 2.33546100 & -2.08095300 & 1.73184700 \\
\hline $\mathrm{H}$ & 2.21152400 & -1.43272700 & 1.00693100 \\
\hline $\mathrm{H}$ & 3.28572800 & -2.09706800 & 1.93502400 \\
\hline $\mathrm{O}$ & 5.02966300 & -1.50168800 & 2.34963100 \\
\hline $\mathrm{H}$ & 4.78886600 & -0.60251800 & 2.66671400 \\
\hline $\mathrm{H}$ & 5.71535100 & -1.84690900 & 2.92746700 \\
\hline $\mathrm{O}$ & 1.45138100 & -3.77599900 & -1.87639100 \\
\hline $\mathrm{H}$ & 0.76089400 & -4.34815100 & -1.49625800 \\
\hline $\mathrm{H}$ & 1.03257500 & -3.27510300 & -2.58822500 \\
\hline $\mathrm{O}$ & 2.30200600 & 1.95633400 & -3.26521600 \\
\hline $\mathrm{H}$ & 2.60245600 & 2.62313300 & -2.62797500 \\
\hline $\mathrm{H}$ & 2.61861500 & 1.08861900 & -2.95968100 \\
\hline $\mathrm{O}$ & 1.55567800 & -0.13202100 & 3.66942800 \\
\hline $\mathrm{H}$ & 0.84735900 & 0.45341800 & 3.34718600 \\
\hline $\mathrm{H}$ & 1.61783500 & -0.87325000 & 3.04383800 \\
\hline $\mathrm{O}$ & 3.95564400 & 0.91998200 & 2.86276000 \\
\hline $\mathrm{H}$ & 3.71910400 & 1.22279500 & 1.97289600 \\
\hline $\mathrm{H}$ & 3.10081900 & 0.64267500 & 3.25341400 \\
\hline $\mathrm{O}$ & -0.15397300 & -1.67517100 & -3.19224500 \\
\hline $\mathrm{H}$ & -0.82680900 & -2.04035100 & -3.77704600 \\
\hline $\mathrm{H}$ & -0.48999500 & -1.74769800 & -2.27529000 \\
\hline $\mathrm{O}$ & -2.76281300 & -3.53817300 & 0.00679000 \\
\hline $\mathrm{H}$ & -3.23143000 & -3.70613800 & 0.83168000 \\
\hline $\mathrm{H}$ & -3.44326700 & -3.30961200 & -0.63586600 \\
\hline $\mathrm{O}$ & 0.37576600 & -4.06946900 & 2.10097600 \\
\hline $\mathrm{H}$ & 0.17984100 & -4.47925200 & 1.24743400 \\
\hline $\mathrm{H}$ & 1.09321200 & -3.43681700 & 1.93608200 \\
\hline $\mathrm{O}$ & -2.41870000 & 2.52771000 & -2.23265900 \\
\hline $\mathrm{H}$ & -2.86326000 & 2.97247200 & -2.95984700 \\
\hline $\mathrm{H}$ & -1.67733400 & 2.02247400 & -2.6357610 \\
\hline $\mathrm{O}$ & -0.53276300 & -5.08982100 & -0.45625700 \\
\hline $\mathrm{H}$ & -1.41771900 & -4.68300700 & -0.40582700 \\
\hline $\mathrm{H}$ & -0.67143900 & -6.03823800 & -0.5440290 \\
\hline $\mathrm{O}$ & 2.54663100 & -0.77690200 & -2.62741400 \\
\hline $\mathrm{H}$ & 2.29593400 & -0.84444900 & -1.69028700 \\
\hline $\mathrm{H}$ & 1.76592400 & -1.10164500 & -3.0968520 \\
\hline $\mathrm{O}$ & -0.35294100 & 1.20555600 & -3.34274700 \\
\hline $\mathrm{H}$ & -0.27580100 & 0.24013000 & -3.3318300 \\
\hline $\mathrm{H}$ & 0.55707700 & 1.55488000 & -3.26560700 \\
\hline $\mathrm{O}$ & 4.11397700 & -3.57512400 & -1.31066700 \\
\hline $\mathrm{H}$ & 4.61158500 & -3.92669100 & -2.0538190 \\
\hline $\mathrm{H}$ & 3.17262100 & -3.68312200 & -1.5457410 \\
\hline
\end{tabular}




$\begin{array}{lrrr}\mathrm{O} & -0.29818000 & 1.73209500 & 2.69757300 \\ \mathrm{H} & -1.07005100 & 1.89524600 & 3.24873400 \\ \mathrm{H} & -0.62597000 & 1.30603000 & 1.86473600 \\ \mathrm{O} & 1.55913600 & 3.90968100 & 2.47501100 \\ \mathrm{H} & 0.91349400 & 3.18503600 & 2.48066100 \\ \mathrm{H} & 1.35533800 & 4.42969700 & 1.68611300\end{array}$

$\begin{array}{lrrr}\left\{(\mathrm{PFH}) \cdot\left(\mathrm{H}_{2} \mathrm{O}\right)_{20}\right\}^{+} & & \\ \mathrm{N} & -1.06919500 & 0.07973600 & 0.32314300 \\ \mathrm{~N} & 1.26886900 & -0.14113400 & -0.05532900 \\ \mathrm{~N} & -0.20489100 & -1.67668500 & -0.87667000 \\ \mathrm{~N} & 2.93987600 & 1.33807200 & 0.39781000 \\ \mathrm{~N} & 0.86599400 & 2.20557500 & -0.09430800 \\ \mathrm{C} & -3.42456700 & 0.49366000 & 0.88098200 \\ \mathrm{C} & -4.84068200 & 0.06580500 & 0.58405600 \\ \mathrm{C} & -2.44152800 & -0.35888000 & 0.08242000 \\ \mathrm{C} & -5.64959200 & 0.80427200 & -0.27986100 \\ \mathrm{C} & -5.34975800 & -1.10974700 & 1.14232200 \\ \mathrm{C} & -6.94099000 & 0.37865300 & -0.58218800 \\ \mathrm{C} & -6.63851000 & -1.53865800 & 0.84201500 \\ \mathrm{C} & -7.43790500 & -0.79484100 & -0.02336000 \\ \mathrm{C} & -0.01668200 & -0.54213700 & -0.19939400 \\ \mathrm{C} & 1.64702900 & 1.13148200 & 0.07457000 \\ \mathrm{H} & -3.28204200 & 1.54856900 & 0.63299300 \\ \mathrm{H} & -3.21335500 & 0.36893900 & 1.94662000 \\ \mathrm{H} & -2.55640000 & -1.40587500 & 0.37621400 \\ \mathrm{H} & -2.65617200 & -0.28379200 & -0.98932300 \\ \mathrm{H} & -5.27068500 & 1.72365000 & -0.71421400 \\ \mathrm{H} & -4.73264900 & -1.68915500 & 1.82257700 \\ \mathrm{H} & -7.55823600 & 0.96579100 & -1.25204100 \\ \mathrm{H} & -7.02049000 & -2.45059900 & 1.28575100 \\ \mathrm{H} & -8.44277700 & -1.12639500 & -0.25639800 \\ \mathrm{H} & 0.61603600 & -2.20073500 & -1.17583300 \\ \mathrm{H} & 3.53689000 & 0.50439600 & 0.37627600 \\ \mathrm{H} & -1.06252600 & -2.21194900 & -0.77477200 \\ \mathrm{H} & 3.35463200 & 2.22233700 & 0.11052400 \\ \mathrm{H} & 1.26402800 & 3.13325800 & -0.01087400 \\ \mathrm{H} & -0.06180800 & 2.12473000 & -0.49622800 \\ \mathrm{O} & -1.09400200 & 5.01825600 & -1.11920600 \\ \mathrm{H} & -1.64421100 & 5.67821300 & -1.55182300 \\ \mathrm{H} & -1.35369100 & 4.14477800 & -1.48153600 \\ \mathrm{O} & 1.50965900 & 5.29575100 & -0.32201800 \\ \mathrm{H} & 0.66726800 & 5.36130200 & -0.81218400 \\ \mathrm{H} & 1.76451700 & 6.19639500 & -0.09480500 \\ \mathrm{O} & 4.47611100 & -1.05128000 & 0.54714700\end{array}$




\begin{tabular}{|c|c|c|c|}
\hline $\mathrm{H}$ & 4.61018100 & -1.27945600 & 1.48068000 \\
\hline $\mathrm{H}$ & 4.39495400 & -1.90427300 & 0.07882700 \\
\hline $\mathrm{O}$ & 3.61145900 & 3.73393600 & -1.11970100 \\
\hline $\mathrm{H}$ & 4.46538200 & 4.16819700 & -1.20758400 \\
\hline $\mathrm{H}$ & 2.95277600 & 4.43449900 & -0.95518700 \\
\hline $\mathrm{O}$ & 1.78323800 & -1.95185900 & 2.08725900 \\
\hline $\mathrm{H}$ & 1.96567400 & -1.43742700 & 1.28049600 \\
\hline $\mathrm{H}$ & 2.62861400 & -1.99822800 & 2.56834100 \\
\hline $\mathrm{O}$ & 4.25104000 & -1.44784900 & 3.30977900 \\
\hline $\mathrm{H}$ & 4.04169000 & -0.51363300 & 3.53154000 \\
\hline $\mathrm{H}$ & 4.78953300 & -1.80825000 & 4.01944900 \\
\hline $\mathrm{O}$ & 1.73450700 & -3.63278300 & -1.84462800 \\
\hline $\mathrm{H}$ & 1.17281000 & -4.40899400 & -1.64342500 \\
\hline $\mathrm{H}$ & 1.73696600 & -3.52831000 & -2.80311300 \\
\hline $\mathrm{O}$ & 2.77066800 & 1.78748800 & -2.98039400 \\
\hline $\mathrm{H}$ & 3.12023400 & 2.45635900 & -2.36909900 \\
\hline $\mathrm{H}$ & 3.10686300 & 0.92134400 & -2.69011200 \\
\hline $\mathrm{O}$ & 0.74308600 & 0.23426000 & 3.60772200 \\
\hline $\mathrm{H}$ & 0.11933700 & 0.84106000 & 3.17456000 \\
\hline $\mathrm{H}$ & 0.85232100 & -0.54502800 & 3.03827000 \\
\hline $\mathrm{O}$ & 3.32601000 & 1.08678700 & 3.47811500 \\
\hline $\mathrm{H}$ & 3.35553100 & 1.39519400 & 2.56255500 \\
\hline $\mathrm{H}$ & 2.37820000 & 0.87762700 & 3.61786700 \\
\hline $\mathrm{O}$ & 0.72210600 & -1.72376300 & -3.88780100 \\
\hline $\mathrm{H}$ & 0.81594400 & -1.73823500 & -4.84745800 \\
\hline $\mathrm{H}$ & -0.08822000 & -2.21214600 & -3.70006500 \\
\hline $\mathrm{O}$ & -1.98819100 & -3.84148700 & -0.03056200 \\
\hline $\mathrm{H}$ & -1.41762400 & -3.86846300 & 0.76636500 \\
\hline $\mathrm{H}$ & -2.88579300 & -4.04488800 & 0.24865900 \\
\hline $\mathrm{O}$ & 0.12895700 & -4.13483200 & 1.62536600 \\
\hline $\mathrm{H}$ & 0.54586500 & -4.71723100 & 0.97718100 \\
\hline $\mathrm{H}$ & 0.75245600 & -3.40103300 & 1.78262000 \\
\hline $\mathrm{O}$ & -1.67911100 & 2.45065700 & -1.73670200 \\
\hline $\mathrm{H}$ & -2.60131500 & 2.23731100 & -1.90479800 \\
\hline $\mathrm{H}$ & -1.13539600 & 1.86663900 & -2.31275700 \\
\hline $\mathrm{O}$ & 0.02300300 & -5.53168800 & -0.91310200 \\
\hline $\mathrm{H}$ & -0.86466600 & -5.14187300 & -0.82141300 \\
\hline $\mathrm{H}$ & -0.09328800 & -6.43455600 & -1.22309800 \\
\hline $\mathrm{O}$ & 3.04909000 & -0.89946300 & -2.27872500 \\
\hline $\mathrm{H}$ & 2.64516600 & -0.87870600 & -1.39554600 \\
\hline $\mathrm{H}$ & 2.34917900 & -1.25358800 & -2.84625700 \\
\hline $\mathrm{O}$ & 0.15497600 & 0.97753000 & -2.95002900 \\
\hline $\mathrm{H}$ & 0.25050000 & 0.06173900 & -3.24862500 \\
\hline $\mathrm{H}$ & 1.05623600 & 1.36133700 & -2.9556800 \\
\hline
\end{tabular}




\begin{tabular}{|c|c|c|c|}
\hline $\mathrm{O}$ & 4.16928400 & -3.55719000 & -0.54916800 \\
\hline $\mathrm{H}$ & 4.85742200 & -3.89574500 & -1.12860200 \\
\hline $\mathrm{H}$ & 3.34118100 & -3.60581800 & -1.06331800 \\
\hline $\mathrm{O}$ & -1.02516300 & 2.06438400 & 2.41620200 \\
\hline $\mathrm{H}$ & -0.80935900 & 2.98396200 & 2.14074400 \\
\hline $\mathrm{H}$ & -1.83141800 & 2.13183700 & 2.93818300 \\
\hline $\mathrm{O}$ & -0.58966100 & 4.61281500 & 1.62601700 \\
\hline $\mathrm{H}$ & -0.98528100 & 4.80465100 & 0.75846400 \\
\hline $\mathrm{H}$ & 0.33011100 & 4.88353000 & 1.51741900 \\
\hline $\mathrm{H}$ & -0.93309000 & 0.85278200 & 0.97653400 \\
\hline $\multicolumn{4}{|c|}{\left(\mathrm{PFH}_{2}\right) \cdot\left(\mathrm{H}_{2} \mathrm{O}\right)_{20}}^{2+}$ \\
\hline $\mathrm{N}$ & 0.64501000 & -0.09564900 & -0.78573600 \\
\hline $\mathrm{N}$ & -1.60300100 & 0.02499500 & -0.12555000 \\
\hline $\mathrm{N}$ & -0.30365800 & -1.74119100 & 0.52334400 \\
\hline $\mathrm{N}$ & -3.14384100 & 1.70091900 & -0.11827600 \\
\hline $\mathrm{N}$ & -0.90277300 & 2.26180000 & -0.05494000 \\
\hline $\mathrm{C}$ & 3.03926800 & 0.39386300 & -1.07045000 \\
\hline $\mathrm{C}$ & 4.41969600 & -0.14498000 & -0.78778900 \\
\hline $\mathrm{C}$ & 1.99788900 & -0.62072300 & -0.60546500 \\
\hline $\mathrm{C}$ & 5.05656600 & 0.15029700 & 0.41897000 \\
\hline $\mathrm{C}$ & 5.05302800 & -0.99241400 & -1.69869500 \\
\hline $\mathrm{C}$ & 6.30712200 & -0.38920600 & 0.70829300 \\
\hline $\mathrm{C}$ & 6.30295300 & -1.53380300 & -1.41118700 \\
\hline $\mathrm{C}$ & 6.93273400 & -1.23322300 & -0.20591400 \\
\hline $\mathrm{C}$ & -0.38192000 & -0.60412700 & -0.13841100 \\
\hline $\mathrm{C}$ & -1.86814600 & 1.35300600 & -0.09639700 \\
\hline $\mathrm{H}$ & 2.88475600 & 1.33536800 & -0.53568100 \\
\hline $\mathrm{H}$ & 2.91656000 & 0.58234600 & -2.13898600 \\
\hline $\mathrm{H}$ & 2.10077400 & -1.56104300 & -1.15518200 \\
\hline $\mathrm{H}$ & 2.13861400 & -0.82509500 & 0.46055800 \\
\hline $\mathrm{H}$ & 4.56385500 & 0.81117500 & 1.12563000 \\
\hline $\mathrm{H}$ & 4.56505600 & -1.22498400 & -2.64023200 \\
\hline $\mathrm{H}$ & 6.79431200 & -0.14874700 & 1.64615100 \\
\hline $\mathrm{H}$ & 6.78588200 & -2.18697900 & -2.12859000 \\
\hline $\mathrm{H}$ & 7.90687800 & -1.65181100 & 0.01764300 \\
\hline $\mathrm{H}$ & -1.05724900 & -2.02731400 & 1.14479000 \\
\hline $\mathrm{H}$ & -3.84370300 & 0.96364600 & -0.13189900 \\
\hline $\mathrm{H}$ & 0.50672700 & -2.35175900 & 0.43063200 \\
\hline $\mathrm{H}$ & -3.39329800 & 2.63358600 & 0.20045600 \\
\hline $\mathrm{H}$ & -1.13265400 & 3.25030000 & -0.00876800 \\
\hline $\mathrm{H}$ & 0.01203100 & 1.98049000 & 0.27805900 \\
\hline
\end{tabular}




\begin{tabular}{|c|c|c|c|}
\hline $\mathrm{O}$ & 1.95713200 & 4.72373900 & 0.88515900 \\
\hline $\mathrm{H}$ & 2.58844100 & 5.41477600 & 1.10830100 \\
\hline $\mathrm{H}$ & 2.26805600 & 3.90223000 & 1.33109100 \\
\hline $\mathrm{O}$ & -0.74242300 & 5.20239500 & 0.54436900 \\
\hline $\mathrm{H}$ & 0.17577300 & 5.14819100 & 0.87537800 \\
\hline $\mathrm{H}$ & -0.89455400 & 6.12603400 & 0.31732100 \\
\hline $\mathrm{O}$ & -4.09853200 & -1.07346600 & -0.09727400 \\
\hline $\mathrm{H}$ & -4.32552500 & -1.48712700 & -0.95131600 \\
\hline $\mathrm{H}$ & -4.32225200 & -1.71224800 & 0.60352400 \\
\hline $\mathrm{O}$ & -2.98407600 & 4.00048400 & 1.59453800 \\
\hline $\mathrm{H}$ & -3.69950800 & 4.54186900 & 1.94247200 \\
\hline $\mathrm{H}$ & -2.24176700 & 4.60261600 & 1.40464200 \\
\hline $\mathrm{O}$ & -1.68580700 & -1.71865200 & -2.50316000 \\
\hline $\mathrm{H}$ & -2.56013400 & -2.11990100 & -2.61118700 \\
\hline $\mathrm{H}$ & -1.01851100 & -2.41363100 & -2.36826600 \\
\hline $\mathrm{O}$ & -4.47698600 & -1.87235100 & -2.70711600 \\
\hline $\mathrm{H}$ & -4.64615300 & -0.96686800 & -3.04702300 \\
\hline $\mathrm{H}$ & -5.14574300 & -2.45050500 & -3.08471400 \\
\hline $\mathrm{O}$ & -1.75431000 & -3.83494200 & 2.00568100 \\
\hline $\mathrm{H}$ & -1.29858000 & -4.42503200 & 1.37489400 \\
\hline $\mathrm{H}$ & -1.52622900 & -4.16370400 & 2.88146300 \\
\hline $\mathrm{O}$ & -1.92474100 & 1.82724100 & 3.07328000 \\
\hline $\mathrm{H}$ & -2.39077900 & 2.54926300 & 2.62151900 \\
\hline $\mathrm{H}$ & -2.39940900 & 0.99761700 & 2.89105300 \\
\hline $\mathrm{O}$ & -1.93514300 & 0.92629700 & -3.11097200 \\
\hline $\mathrm{H}$ & -1.10563400 & 1.42891800 & -3.10310300 \\
\hline $\mathrm{H}$ & -1.70799200 & -0.00469100 & -2.92496500 \\
\hline $\mathrm{O}$ & -4.60561100 & 0.76277200 & -3.34943300 \\
\hline $\mathrm{H}$ & -5.11518500 & 1.33504000 & -2.76938000 \\
\hline $\mathrm{H}$ & -3.66746000 & 1.02345100 & -3.24510400 \\
\hline $\mathrm{O}$ & 0.06700500 & -1.49161200 & 3.72938600 \\
\hline $\mathrm{H}$ & 0.22313700 & -1.33166500 & 4.66715100 \\
\hline $\mathrm{H}$ & 0.48849200 & -2.33757700 & 3.54219800 \\
\hline $\mathrm{O}$ & 2.03277300 & -3.62693500 & 0.74138700 \\
\hline $\mathrm{H}$ & 2.66961000 & -3.66724900 & 0.01840300 \\
\hline $\mathrm{H}$ & 2.55925000 & -3.55226100 & 1.54524200 \\
\hline $\mathrm{O}$ & 0.42156800 & -3.41224600 & -1.91815300 \\
\hline $\mathrm{H}$ & 0.15459000 & -4.14278800 & -1.33366100 \\
\hline $\mathrm{H}$ & 0.91333700 & -3.81701400 & -2.63941900 \\
\hline $\mathrm{O}$ & 2.71840700 & 2.37869800 & 1.98961100 \\
\hline $\mathrm{H}$ & 3.18879400 & 2.42116900 & 2.82751900 \\
\hline
\end{tabular}




$\begin{array}{lrrr}\mathrm{H} & 1.94022200 & 1.80075600 & 2.14294900 \\ \mathrm{O} & -0.14350700 & -5.17521600 & 0.19949200 \\ \mathrm{H} & 0.73587300 & -4.94957400 & 0.55174800 \\ \mathrm{H} & -0.17606800 & -6.13301900 & 0.10615400 \\ \mathrm{O} & -2.44272500 & -0.83101800 & 2.59388400 \\ \mathrm{H} & -1.73198200 & -1.20299300 & 3.13997000 \\ \mathrm{H} & -3.19416700 & -1.43976100 & 2.66016100 \\ \mathrm{O} & 0.46922900 & 0.88299700 & 2.18205000 \\ \mathrm{H} & 0.49371200 & 0.02313500 & 2.63239700 \\ \mathrm{H} & -0.33184500 & 1.32599200 & 2.53838000 \\ \mathrm{O} & -4.29276100 & -2.84517900 & 2.01260500 \\ \mathrm{H} & -5.02755300 & -3.35324900 & 2.36737200 \\ \mathrm{H} & -3.51786900 & -3.43712300 & 1.95979200 \\ \mathrm{O} & 0.62645000 & 1.96039100 & -2.78758300 \\ \mathrm{H} & 0.81060000 & 2.85367700 & -2.41597000 \\ \mathrm{H} & 1.20188000 & 1.85654500 & -3.55220200 \\ \mathrm{O} & 1.05330400 & 4.40118400 & -1.74455500 \\ \mathrm{H} & 1.53295800 & 4.47326100 & -0.89952100 \\ \mathrm{H} & 0.21113400 & 4.83640800 & -1.57038100 \\ \mathrm{H} & 0.50726400 & 0.64560900 & -1.48160800 \\ \mathrm{H} & -2.43803800 & -0.58266000 & -0.03305000\end{array}$


Table S14. Cartesian coordinates corresponding to the optimized structures of $\left\{(\mathrm{PB}) \cdot\left(\mathrm{H}_{2} \mathrm{O}\right)_{20}\right\}$, $\left\{(\mathrm{PBH}) \cdot\left(\mathrm{H}_{2} \mathrm{O}\right)_{20}\right\}^{+}$and $\left\{\left(\mathrm{PBH}_{2}\right) \cdot\left(\mathrm{H}_{2} \mathrm{O}\right)_{20}\right\}^{2+}$ under study.

$\begin{array}{lrrr}\left\{(\mathrm{PB}) \cdot\left(\mathrm{H}_{2} \mathrm{O}\right)_{20}\right\} & & \\ \mathrm{C} & -1.19753100 & 0.21748100 & -0.95498400 \\ \mathrm{~N} & -1.99212100 & 0.37272500 & -2.06017900 \\ \mathrm{~N} & 0.05137300 & 0.75163500 & -1.13147200 \\ \mathrm{C} & 1.07868700 & 0.49051800 & -0.31760600 \\ \mathrm{~N} & 1.02502600 & -0.24177100 & 0.80266700 \\ \mathrm{H} & 1.87480300 & -0.57670100 & 1.24971800 \\ \mathrm{~N} & 2.26831200 & 1.00004000 & -0.67797900 \\ \mathrm{~N} & -1.60345300 & -0.38410000 & 0.13484100 \\ \mathrm{C} & -2.83487900 & -1.07218000 & 0.13817000 \\ \mathrm{C} & -3.04778800 & -2.17280600 & -0.70596900 \\ \mathrm{C} & -3.82430300 & -0.74342700 & 1.07278200 \\ \mathrm{C} & -4.22292400 & -2.90858300 & -0.62785700 \\ \mathrm{H} & -2.27430400 & -2.43824400 & -1.41787600 \\ \mathrm{C} & -4.99757400 & -1.49041100 & 1.15171100 \\ \mathrm{H} & -3.66632400 & 0.10147100 & 1.73274900 \\ \mathrm{C} & -5.20590000 & -2.57215300 & 0.30270300 \\ \mathrm{H} & -4.36882000 & -3.75473000 & -1.28965300 \\ \mathrm{H} & -5.75326300 & -1.21855900 & 1.87984000 \\ \mathrm{H} & -6.11920900 & -3.15117100 & 0.36657100 \\ \mathrm{H} & 3.03030700 & 1.00194300 & -0.00053200 \\ \mathrm{H} & -3.00025300 & 0.32683700 & -1.93796000 \\ \mathrm{H} & 2.26044900 & 1.68194800 & -1.42802200 \\ \mathrm{H} & 0.16316400 & -0.74863600 & 0.96743400 \\ \mathrm{H} & -1.67942400 & 1.08180900 & -2.70728300 \\ \mathrm{O} & -0.87656100 & -2.47575700 & 2.35067000 \\ \mathrm{H} & -0.45256400 & -2.74767200 & 1.51266600 \\ \mathrm{H} & -1.74931400 & -2.14711200 & 2.10173800 \\ \mathrm{O} & 0.44233500 & -0.87089900 & 4.06001700 \\ \mathrm{H} & 0.21363500 & -1.04792400 & 4.97834700 \\ \mathrm{H} & -0.03406200 & -1.53258500 & 3.50560600 \\ \mathrm{O} & 0.16371800 & -2.29591100 & -2.67570000 \\ \mathrm{H} & -0.55097800 & -2.70752800 & -3.17256600 \\ \mathrm{H} & 0.30644300 & -1.41713800 & -3.08588800 \\ \mathrm{O} & 0.92912900 & 0.17536800 & -3.62438400 \\ \mathrm{H} & 0.62472600 & 0.61926800 & -2.78845400 \\ \mathrm{H} & 0.58047700 & 0.67991400 & -4.36548400 \\ \mathrm{O} & -4.91272600 & 1.02127900 & -1.70112100 \\ \mathrm{H} & -5.35498500 & 1.49786300 & -2.40884500 \\ & -4.49075200 & 1.71046700 & -1.15209300 \\ \mathrm{H} & -3.43046400 & 2.95072500 & -0.41319400\end{array}$




$\begin{array}{lrrr}\mathrm{H} & -3.10147600 & 3.07268700 & 0.49812000 \\ \mathrm{H} & -2.62765200 & 3.05983100 & -0.94587200 \\ \mathrm{O} & -0.75131100 & 3.54606300 & -1.25191300 \\ \mathrm{H} & -0.73417500 & 4.45239300 & -0.92292400 \\ \mathrm{H} & -0.35722100 & 3.01018500 & -0.53775500 \\ \mathrm{O} & 2.66390500 & 4.26762200 & -0.19121200 \\ \mathrm{H} & 2.80861200 & 5.21731700 & -0.15414900 \\ \mathrm{H} & 2.45096100 & 4.06250800 & -1.11884800 \\ \mathrm{O} & 1.67943900 & 3.23759000 & -2.56905300 \\ \mathrm{H} & 1.79159500 & 3.41863200 & -3.50648200 \\ \mathrm{H} & 0.72698900 & 3.30526800 & -2.37046700 \\ \mathrm{O} & 5.19061300 & -1.63884800 & 1.66330100 \\ \mathrm{H} & 6.09083400 & -1.97257600 & 1.63791100 \\ \mathrm{H} & 4.70054200 & -2.03998000 & 0.91470700 \\ \mathrm{O} & 1.84997500 & 1.43466900 & 3.23456300 \\ \mathrm{H} & 1.97314500 & 1.95993800 & 4.03235800 \\ \mathrm{H} & 1.38976900 & 0.62630900 & 3.52067200 \\ \mathrm{O} & 4.07531000 & 0.94309100 & 1.56212700 \\ \mathrm{H} & 4.53599000 & 0.10008500 & 1.70343900 \\ \mathrm{H} & 3.41516200 & 1.03307400 & 2.26858400 \\ \mathrm{O} & 2.61685400 & -1.83924200 & 2.59068900 \\ \mathrm{H} & 2.20456300 & -1.59598600 & 3.42892300 \\ \mathrm{H} & 3.57548600 & -1.85684700 & 2.71755400 \\ \mathrm{H} & 3.36188000 & -2.53284700 & -0.08811200 \\ \mathrm{O} & 2.49350800 & -2.62279900 & 0.33036800 \\ \mathrm{H} & 3.23628500 & -2.81398500 & -1.00978000 \\ \mathrm{H} & 0.46336200 & -3.14869700 & 0.01438100 \\ \mathrm{H} & 0.25236100 & -2.69961500 & -0.82467200 \\ \mathrm{O} & 0.44039300 & -4.08912700 & -0.19397500 \\ \mathrm{H} & -1.54366200 & 0.79756400 & 2.73911500 \\ \mathrm{H} & -0.83911600 & 0.43479400 & 3.29148600 \\ \mathrm{O} & -1.41947000 & 0.41174800 & 1.84709000 \\ \mathrm{H} & -2.08822800 & 3.37477000 & 1.99770400 \\ \mathrm{H} & -1.18065100 & 3.55796600 & 1.70503500 \\ \mathrm{O} & -1.98531000 & 2.49770900 & 2.41270300 \\ \mathrm{H} & 0.59089400 & 3.06268900 & 1.19868500 \\ \mathrm{H} & 1.38047400 & 3.50131400 & 0.82871200 \\ \mathrm{H} & 0.91993000 & 2.38919700 & 1.81840100 \\ \mathrm{H} & 3.79493000 & -1.60741900 & -3.44606100 \\ \mathrm{H} & & -0.70200800 & -3.75320100 \\ \mathrm{H} & 2.75488100 & -3.24576600 & -2.72735400 \\ \mathrm{H} & -36800 & -4.11217300 & -3.10767200 \\ \mathrm{H} & -3.08822100 & -2.80179500 \\ \mathrm{H} & & & \\ \mathrm{H} & & & \end{array}$




\begin{tabular}{|c|c|c|c|}
\hline $\multicolumn{4}{|c|}{(\mathrm{PBH}) \cdot\left(\mathrm{H}_{2} \mathrm{O}\right)_{20}}^{+}$ \\
\hline $\mathrm{C}$ & 1.05508000 & -0.06621400 & -1.18387600 \\
\hline $\mathrm{N}$ & 1.64086800 & 0.24619200 & -2.34234700 \\
\hline $\mathrm{N}$ & -0.13839000 & -0.66168000 & -1.23849100 \\
\hline $\mathrm{C}$ & -1.06360000 & -0.60297000 & -0.25663700 \\
\hline $\mathrm{N}$ & -1.01991300 & 0.20718500 & 0.80163200 \\
\hline $\mathrm{H}$ & -1.83982700 & 0.33818900 & 1.39202400 \\
\hline $\mathrm{N}$ & -2.12375100 & -1.39033500 & -0.41064100 \\
\hline $\mathrm{N}$ & 1.71452200 & 0.19173200 & -0.03581300 \\
\hline $\mathrm{C}$ & 2.84017300 & 1.05236800 & 0.09275100 \\
\hline $\mathrm{C}$ & 2.81891600 & 2.33190000 & -0.46579000 \\
\hline $\mathrm{C}$ & 3.92878900 & 0.63600100 & 0.85460100 \\
\hline $\mathrm{C}$ & 3.89850600 & 3.18554500 & -0.27076700 \\
\hline $\mathrm{H}$ & 1.95860300 & 2.65420500 & -1.04263500 \\
\hline $\mathrm{C}$ & 4.99903100 & 1.50243100 & 1.05415500 \\
\hline $\mathrm{H}$ & 3.92656900 & -0.35814900 & 1.28537200 \\
\hline $\mathrm{C}$ & 4.99069700 & 2.77463700 & 0.49017700 \\
\hline $\mathrm{H}$ & 3.87889800 & 4.17760200 & -0.70544500 \\
\hline $\mathrm{H}$ & 5.84373800 & 1.17726000 & 1.64951900 \\
\hline $\mathrm{H}$ & 5.82726600 & 3.44477400 & 0.64567000 \\
\hline $\mathrm{H}$ & 1.43094600 & -0.28460400 & 0.82479700 \\
\hline $\mathrm{H}$ & -2.82579900 & -1.44872800 & 0.32989900 \\
\hline $\mathrm{H}$ & 2.64623000 & 0.41613300 & -2.41119700 \\
\hline $\mathrm{H}$ & -2.09128700 & -2.07537300 & -1.15797600 \\
\hline $\mathrm{H}$ & -0.29589300 & 0.90777200 & 0.90309100 \\
\hline $\mathrm{H}$ & 1.12678100 & 0.05822600 & -3.18931500 \\
\hline $\mathrm{O}$ & 0.46633400 & 2.57743300 & 1.79220600 \\
\hline $\mathrm{H}$ & -0.11957800 & 2.98305600 & 1.12315200 \\
\hline $\mathrm{H}$ & 1.37045300 & 2.80694700 & 1.55000100 \\
\hline $\mathrm{O}$ & -0.14401400 & 1.14345800 & 4.01156500 \\
\hline $\mathrm{H}$ & 0.04117500 & 1.55239300 & 4.86308000 \\
\hline $\mathrm{H}$ & 0.07491600 & 1.79946300 & 3.31660600 \\
\hline $\mathrm{O}$ & -1.16151600 & 2.30076200 & -2.85852100 \\
\hline $\mathrm{H}$ & -0.54503000 & 2.80776300 & -3.39734400 \\
\hline $\mathrm{H}$ & -1.16443300 & 1.39528800 & -3.22752400 \\
\hline $\mathrm{O}$ & -1.38685300 & -0.34925700 & -3.63930200 \\
\hline $\mathrm{H}$ & -0.94582000 & -0.74704500 & -2.85234000 \\
\hline $\mathrm{H}$ & -1.15898100 & -0.88021100 & -4.40899000 \\
\hline $\mathrm{O}$ & 4.53759100 & 0.17901800 & -2.48435300 \\
\hline $\mathrm{H}$ & 4.94197100 & -0.12196100 & -3.30307300 \\
\hline $\mathrm{H}$ & 4.46303600 & -0.61668200 & -1.92079700 \\
\hline $\mathrm{O}$ & 3.90881100 & -2.08332600 & -1.06815100 \\
\hline $\mathrm{H}$ & 3.79496400 & -2.19684700 & -0.10965300 \\
\hline $\mathrm{H}$ & 600 & -2.42432000 & -1.4 \\
\hline
\end{tabular}




\begin{tabular}{|c|c|c|c|}
\hline $\mathrm{O}$ & 1.33007700 & -3.26248600 & -1.33986500 \\
\hline $\mathrm{H}$ & 1.57057200 & -4.16685500 & -1.10590400 \\
\hline $\mathrm{H}$ & 0.98708500 & -2.87230900 & -0.51392800 \\
\hline $\mathrm{O}$ & -1.51099900 & -4.50963400 & 0.30104500 \\
\hline $\mathrm{H}$ & -1.40732700 & -5.46169300 & 0.38755300 \\
\hline $\mathrm{H}$ & -1.54086600 & -4.32747800 & -0.65432200 \\
\hline $\mathrm{O}$ & -1.27992200 & -3.46169900 & -2.29193700 \\
\hline $\mathrm{H}$ & -1.46906200 & -3.83404600 & -3.15807300 \\
\hline $\mathrm{H}$ & -0.31515700 & -3.33069600 & -2.23558300 \\
\hline $\mathrm{O}$ & -5.24641500 & 0.86161800 & 2.17030500 \\
\hline $\mathrm{H}$ & -6.18111500 & 1.05766900 & 2.27192500 \\
\hline $\mathrm{H}$ & -4.94415100 & 1.25711700 & 1.32484500 \\
\hline $\mathrm{O}$ & -1.28191100 & -1.45021000 & 3.38747300 \\
\hline $\mathrm{H}$ & -1.20348100 & -1.98734000 & 4.18281600 \\
\hline $\mathrm{H}$ & -0.89435800 & -0.58974200 & 3.61867800 \\
\hline $\mathrm{O}$ & -3.69363600 & -1.47161200 & 1.95989600 \\
\hline $\mathrm{H}$ & -4.30145900 & -0.73682900 & 2.14274900 \\
\hline $\mathrm{H}$ & -2.96567600 & -1.40752300 & 2.60090100 \\
\hline $\mathrm{O}$ & -2.64192700 & 1.52106400 & 2.73167700 \\
\hline $\mathrm{H}$ & -2.12629000 & 1.43328400 & 3.54229500 \\
\hline $\mathrm{H}$ & -3.57581400 & 1.39585500 & 2.95366200 \\
\hline $\mathrm{O}$ & -3.84085800 & 1.76015400 & 0.07787800 \\
\hline $\mathrm{H}$ & -2.99574100 & 2.14015800 & 0.36015800 \\
\hline $\mathrm{H}$ & -3.97768600 & 2.09714400 & -0.82284600 \\
\hline $\mathrm{O}$ & -1.31682500 & 3.20639500 & -0.19355300 \\
\hline $\mathrm{H}$ & -1.15181600 & 2.82100500 & -1.07438300 \\
\hline $\mathrm{H}$ & -1.57765000 & 4.11885500 & -0.35885700 \\
\hline $\mathrm{O}$ & 1.54061600 & -0.62374000 & 2.65821600 \\
\hline $\mathrm{H}$ & 2.34394200 & -1.08666300 & 2.92244700 \\
\hline $\mathrm{H}$ & 1.26108000 & -0.03879900 & 3.37582100 \\
\hline $\mathrm{O}$ & 3.12796900 & -2.65207500 & 1.61987200 \\
\hline $\mathrm{H}$ & 3.54951900 & -3.44257700 & 1.97107500 \\
\hline $\mathrm{H}$ & 2.17547900 & -2.85910600 & 1.55411400 \\
\hline $\mathrm{O}$ & 0.37401100 & -2.74634200 & 1.26054200 \\
\hline $\mathrm{H}$ & -0.34556300 & -3.38408800 & 1.08479500 \\
\hline $\mathrm{H}$ & 0.04437800 & -2.11181900 & 1.92052400 \\
\hline $\mathrm{O}$ & -4.24360100 & -0.09626900 & -3.28178700 \\
\hline $\mathrm{H}$ & -4.28342000 & 0.84860700 & -3.07716200 \\
\hline $\mathrm{H}$ & -3.29770600 & -0.27132800 & -3.39400600 \\
\hline $\mathrm{O}$ & -3.88955200 & 2.67351900 & -2.57783000 \\
\hline $\mathrm{H}$ & -4.34158600 & 3.44501100 & -2.93187700 \\
\hline $\mathrm{H}$ & -2.94704400 & 2.76101700 & -2.80931600 \\
\hline
\end{tabular}




\begin{tabular}{|c|c|c|c|}
\hline $\mathrm{C}$ & -0.64315600 & 0.94984000 & -1.14504500 \\
\hline $\mathrm{N}$ & -1.19634700 & 1.62271000 & -2.12555600 \\
\hline $\mathrm{N}$ & 0.72686100 & 0.90148700 & -1.09595200 \\
\hline $\mathrm{C}$ & 1.50757200 & -0.11551200 & -0.58674500 \\
\hline $\mathbf{N}$ & 0.99470700 & -1.24975900 & -0.16851900 \\
\hline $\mathrm{H}$ & 1.58835100 & -1.96626600 & 0.26326300 \\
\hline $\mathrm{N}$ & 2.80532300 & 0.11913900 & -0.55212300 \\
\hline $\mathrm{N}$ & -1.33128600 & 0.34756600 & -0.17658100 \\
\hline 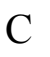 & -2.74480400 & 0.22045300 & -0.16604500 \\
\hline $\mathrm{C}$ & -3.41212400 & -0.30541200 & -1.26940500 \\
\hline $\mathrm{C}$ & -3.44148700 & 0.60674300 & 0.97555400 \\
\hline $\mathrm{C}$ & -4.80027200 & -0.38369900 & -1.24853800 \\
\hline $\mathrm{H}$ & -2.84766100 & -0.64039800 & -2.13111400 \\
\hline $\mathrm{C}$ & -4.82825100 & 0.50140800 & 0.99741700 \\
\hline $\mathrm{H}$ & -2.89979100 & 0.99535800 & 1.83062600 \\
\hline$C$ & -5.51242100 & 0.02391600 & -0.11970500 \\
\hline $\mathrm{H}$ & -5.32579400 & -0.77579200 & -2.11048800 \\
\hline $\mathrm{H}$ & -5.37403800 & 0.80487300 & 1.88202400 \\
\hline $\mathrm{H}$ & -6.59331400 & -0.04029600 & -0.10788000 \\
\hline $\mathrm{H}$ & -0.85359500 & 0.34519000 & 0.73497700 \\
\hline $\mathrm{H}$ & 3.44483800 & -0.66625600 & -0.42676000 \\
\hline $\mathrm{H}$ & -2.15995500 & 1.96110900 & -2.03012700 \\
\hline $\mathrm{H}$ & 3.18642000 & 1.02420800 & -0.83311000 \\
\hline $\mathrm{H}$ & 0.01631900 & -1.49815200 & -0.31099600 \\
\hline $\mathrm{H}$ & -0.58939900 & 2.04016300 & -2.82652600 \\
\hline $\mathrm{O}$ & -1.30279900 & -2.76866800 & 0.39753000 \\
\hline $\mathrm{H}$ & -0.96317300 & -3.68059500 & 0.39036800 \\
\hline $\mathrm{H}$ & -2.27681500 & -2.82143200 & 0.43567900 \\
\hline $\mathrm{O}$ & -0.41849600 & -1.83936100 & 2.89155500 \\
\hline $\mathrm{H}$ & -0.42653500 & -0.87882900 & 2.75497900 \\
\hline $\mathrm{H}$ & -0.74522100 & -2.21244600 & 2.05132700 \\
\hline $\mathrm{O}$ & -0.34218300 & -1.33991200 & -2.81671000 \\
\hline $\mathrm{H}$ & -0.74442900 & -1.04695500 & -3.64077200 \\
\hline $\mathrm{H}$ & 0.59881900 & -1.46810100 & -3.02105500 \\
\hline $\mathrm{O}$ & 1.27686600 & 2.76002300 & -3.07647300 \\
\hline $\mathrm{H}$ & 1.86397200 & 2.66481000 & -3.83274400 \\
\hline $\mathrm{H}$ & 1.54035400 & 3.57260600 & -2.60373800 \\
\hline $\mathrm{O}$ & -3.55599000 & 3.03082800 & -1.33651800 \\
\hline $\mathrm{H}$ & -4.36127400 & 2.58533300 & -1.05243200 \\
\hline $\mathrm{H}$ & -3.04438700 & 3.19843700 & -0.52474000 \\
\hline $\mathrm{O}$ & -1.52316300 & 3.28883600 & 0.55879400 \\
\hline
\end{tabular}




\begin{tabular}{|c|c|c|c|}
\hline $\mathrm{H}$ & -1.58101100 & 3.29924600 & 1.52897100 \\
\hline $\mathrm{H}$ & -1.00178500 & 4.06637400 & 0.33086100 \\
\hline $\mathrm{O}$ & 2.08419400 & 4.53747600 & -1.18004600 \\
\hline $\mathrm{H}$ & 2.10668900 & 5.49737100 & -1.12389000 \\
\hline $\mathrm{H}$ & 1.73476500 & 4.19624000 & -0.32619100 \\
\hline $\mathrm{O}$ & 4.01536300 & 2.29080100 & 1.62993700 \\
\hline $\mathrm{H}$ & 4.64120200 & 2.74675100 & 2.20153900 \\
\hline $\mathrm{H}$ & 4.31110700 & 2.43902800 & 0.71354200 \\
\hline $\mathrm{O}$ & 4.07998000 & 2.65265000 & -1.09599400 \\
\hline $\mathrm{H}$ & 4.80448900 & 2.67116400 & -1.72944000 \\
\hline $\mathrm{H}$ & 3.54373600 & 3.45397500 & -1.24927200 \\
\hline $\mathrm{O}$ & 4.12266600 & -2.41661000 & -0.86853500 \\
\hline $\mathrm{H}$ & 5.04743900 & -2.54837600 & -1.09808800 \\
\hline $\mathrm{H}$ & 3.60376800 & -2.51325600 & -1.69420900 \\
\hline $\mathrm{O}$ & 2.16975500 & -2.36336300 & 3.45842900 \\
\hline $\mathrm{H}$ & 2.33486700 & -2.73082700 & 4.33169400 \\
\hline $\mathrm{H}$ & 1.19460400 & -2.26013900 & 3.36128900 \\
\hline $\mathrm{O}$ & 0.31885600 & -5.01190200 & 0.28993000 \\
\hline $\mathrm{H}$ & 2.55653600 & -3.19020200 & 1.92189300 \\
\hline $\mathrm{H}$ & 0.26547000 & -5.91097400 & 0.62605000 \\
\hline $\mathrm{O}$ & 2.41691100 & -3.45550700 & 0.99298700 \\
\hline $\mathrm{H}$ & 1.15106700 & -4.61700200 & 0.62284000 \\
\hline $\mathrm{H}$ & 3.24139400 & -3.31624600 & 0.49651200 \\
\hline $\mathrm{O}$ & 2.30519900 & -2.49096900 & -2.92074800 \\
\hline $\mathrm{H}$ & 1.67515500 & -3.22463200 & -2.73346700 \\
\hline $\mathrm{H}$ & 2.65100300 & -2.64852700 & -3.80580500 \\
\hline $\mathrm{O}$ & 0.22184300 & -4.08149900 & -2.32526500 \\
\hline $\mathrm{H}$ & -0.39358500 & -3.33599300 & -2.27264200 \\
\hline $\mathrm{H}$ & 0.20772700 & -4.51905000 & -1.45662100 \\
\hline $\mathrm{O}$ & 0.02812300 & 0.88583900 & 2.18977900 \\
\hline $\mathrm{H}$ & -0.37692400 & 1.54345600 & 2.78133600 \\
\hline $\mathrm{H}$ & 0.95902900 & 0.71646200 & 2.49021700 \\
\hline $\mathrm{O}$ & -1.36179700 & 3.00510900 & 3.37476900 \\
\hline $\mathrm{H}$ & -2.12583600 & 2.86081600 & 3.94445800 \\
\hline $\mathrm{H}$ & -0.84447400 & 3.69506100 & 3.80605400 \\
\hline $\mathrm{O}$ & 1.42382200 & 3.30240900 & 1.12093600 \\
\hline $\mathrm{H}$ & 2.26413900 & 2.94822500 & 1.46262900 \\
\hline $\mathrm{H}$ & 0.78726200 & 2.57450300 & 1.15838500 \\
\hline $\mathrm{O}$ & -4.04496300 & -2.95109300 & 0.74005000 \\
\hline $\mathrm{H}$ & -4.59506300 & -2.22392000 & 0.42489700 \\
\hline $\mathrm{H}$ & -4.28507700 & -3.06897800 & 1.66467500 \\
\hline
\end{tabular}


$\mathrm{O}$

$\mathrm{H}$

$\mathrm{H}$ $\begin{array}{llll}2.49514300 & 0.34497000 & 2.88809700\end{array}$

$\begin{array}{lll}3.20749100 & 0.74724700 & 2.36741500\end{array}$

$\begin{array}{llll}2.66973800 & -0.60016100 & 3.02697900\end{array}$

$\begin{array}{llll}1.19159200 & 1.54091400 & -1.75720600\end{array}$ 